\title{
A DECOMPOSITION THEORY FOR UNITARY REPRESENTATIONS OF LOCALLY COMPACT GROUPS
}

\author{
BY \\ JOHN A. ERNEST(1)
}

Introduction. In the classical theory of finite dimensional representations of compact groups, every representation may be expressed uniquely as a direct sum of irreducible representations. This reduces the problem of determining the complete representation theory of such a group to the much simpler problem of determining the irreducible representations. In the past decade or so many attempts have been made to generalize this situation to a theory of (not necessarily finite dimensional) unitary representations of separable locally compact groups. For this purpose, the von Neumann concept of "direct-integrals" of weakly-closed *-algebras of operators [19] was adapted to give a "direct-integral" of representations, which appears to be the natural extension of the concept of a direct sum of representations [17]. There is a natural "duality" between representation theory and the theory of von Neumann algebras. In particular, there is an intrinsic way of classifying representations into types I, II and III, which is equivalent to the Murrayvon Neumann classification of the $W^{*}$-rings of operators generated by the range of the representations. A group is called type $\mathrm{I}$ if all its representations are type I. (Cf. $[1 ; 12 ; 14]$.)

In the case of type I groups, a completely satisfactory decomposition theory is obtained. (Cf. [15] with added amendments [2;6;7 and 8].) We assume throughout this paragraph that the group $G$ is type I. The dual object is then, as in the classical case, the collection $\hat{G}$ of all unitary equivalence classes of irreducible representations of the group $G$, in which a $\sigma$-ring of subsets is specified. This $\sigma$-ring of distinguished sets is called a Borel structure for $\hat{G}$. $\hat{G}$ may also be given a natural "hull-kernel" topology whose closed sets generate the Borel structure for $\hat{G}$. (Cf. $[5 ; 6]$.) A representation $L$ is said to be multiplicity free if its commuting algebra $R(L, L)$ (the ring of operators which commute with every element of the range of $L$ ) is Abelian. Every type I representation can be expressed uniquely (up to order of terms and unitary equivalence) as a discrete direct sum of multiplicity free representations $[14$, Theorem 1.4]. Finally, every multiplicity free representation can be expressed uniquely (up to unitary equivalence and sets of measure zero) as a direct integral of irreducible representations with respect to a $\sigma$-finite measure $\mu$ on $\hat{G}$. The measure $\mu$ is determined only up to absolute continuity. In this way a

Received by the editors September 11, 1961.

(1) National Science Foundation fellow. 
one-to-one order preserving correspondence is set up between the collection of all unitary equivalence classes of multiplicity free representations of a type I group and the collection of all $\sigma$-finite measure classes on $\hat{G},[15$, corollary to Theorem 10.6]. The theory as developed in [15] required an additional technical assumption on the Borel structure in $\hat{G}$. The final touch was put on the type I theory when Glimm [7] proved that this additional hypothesis (that $\hat{G}$ be "smooth") is equivalent to the property that $G$ be type I. (Also see [2].)

However when one leaves the frontiers of the type I case, the analogies with the classical theory of finite dimensional representations break down rapidly. Type II and III representations have no irreducible subrepresentations. Multiplicity theory (see [14]) assumes a more difficult, albeit more interesting, form. Representations can still be decomposed into direct integrals of irreducible representations, but examples were soon discovered [13, Theorem 11] where a type II representation may be expressed as a direct integral of irreducible representations in two different ways, so that no component of one decomposition is unitary equivalent to any component in the second decomposition. Further there is no hope of expressing a nontype $I$ representation as a discrete direct sum of multiplicity free representations, as every multiplicity free representation is type I. Further it has been shown [7] that the Borel structure in the dual $\hat{G}$ is necessarily bad (specifically it does not have a countable separating family of Borel sets) in the nontype I case. As we shall see, all of these difficulties combine to force one to consider a new "dual-object." To continue the narrative it is necessary at this point to define some terms.

Let $x \rightarrow L_{x}$ and $x \rightarrow M_{x}$ denote two unitary representations of a separable locally compact group $G$ over separable Hilbert spaces $\mathfrak{H C}(L)$ and $\mathfrak{H C}(M)$ respectively. $L$ and $M$ are said to be disjoint, denoted $L \circlearrowleft M$, if no subrepresentation of $L$ is equivalent to any subrepresentation of $M$. We say $L$ covers $M$, denoted $L\} M$, if no subrepresentation of $M$ is disjoint from $L$. We say $L$ is quasi-equivalent to $M$, denoted $L \sim M$, if $L$ covers $M$ and $M$ covers $L$. The term "quasi-equivalence" is perhaps misleading as this relation is a bonafide equivalence relation. The adjective "quasi" is used only to distinguish the relation from the more traditional concept of unitary equivalence. As we shall see later, the collection of all quasi-equivalence classes of representations, under the covering relation defined above, forms a $\sigma$-complete, distributive partially ordered system in which every pair of elements are either disjoint or have a greatest lower bound.

If a group is type $I$, it is well known that every quasi-equivalence class contains a unique (up to unitary equivalence) multiplicity free representation. Thus the classification, in the type I case described above, of all multiplicity free representations by means of measure classes on $\hat{G}$, may be interpreted as an order-preserving classification of the quasi-equivalence classes of representations of $G$. Furthermore, a multiplicity theory has been developed, 
not dependent on the type I hypothesis, for distinguishing representations (up to unitary equivalence) within a quasi-equivalence class. (For example see $[14$, Theorem 1.5].) Thus the problem of classifying all representations, up to unitary equivalence, is reduced to that of classifying the quasi-equivalence classes. By analogy with the type I case, we plan to effect this classification by means of a decomposition theory, with each quasi-equivalence class corresponding to a measure class on a dual-object, the points of this dual-object representing the "building blucks."

Let $Q$ denote the collection of all quasi-equivalence classes of representations of some group $G$, partially ordered by the covering relation defined above. The elements of $Q$ which are minimal with respect to this partial ordering we shall call primary classes. We define $\tilde{G}$, the quasi-dual of $G$, to be the collection of these primary classes. In our general decomposition theory, the primary classes will serve as the "building blocks" and the quasidual $\widetilde{G}$ as our "dual object." A representation will be called primary if it is contained in a primary class. Then a representation is primary if and only if it cannot be expressed as the direct sum of two disjoint representations. Let $a(L)$ denote the von Neumann algebra of operators generated by the range of the representation $L$. Then $L$ is primary if and only if $Q(L)$ is a factor in the Murray-von Neumann terminology, i.e., if and only if $Q(L)$ has trivial center. For this reason primary representations are often referred to in the literature as "factor representations." (For example in [14 and 18].) However the term "primary," used in [12] and [16], seems to be more appropriate in the context of group representations and will be used exclusively throughout this paper. By means of the Murray-von Neumann theory of factors, every primary representation, and thus every primary class, may be classified as being either of type I, II or III. (Cf. [12 and 14].)

A $\sigma$-ring of sets, called Borel sets, is specified in $\widetilde{G}$, which will hereafter be called a "Borel structure" for $\tilde{G}$, (cf. [15]). Our main result on decomposition theory (Theorem 2) is that the central decomposition [14, p. 201], often called the "canonical decomposition," may always be taken over $\tilde{G}$. More specifically, to each representation $L$ of $G$, there corresponds a measure $\mu$ on $\tilde{G}$ and a $\mu$-measurable map $y \rightarrow L^{\nu}$ such that $L^{y} \in y$ for all $y \in \tilde{G}, L \simeq \int_{\tilde{G}} L^{\nu} d \mu(y)$ and the range of the projection valued measure associated with this decomposition, is the collection of all projections in the center of $R(L, L)$. Using this result, we obtain a one-to-one order preserving correspondence between $Q$ and a lattice ideal $\operatorname{err}(\tilde{G})$ of measure classes on $\tilde{G}$, (Theorems 3 and 4 ). We call $\operatorname{ern}(\tilde{G})$ the canonical measure lattice on $\tilde{G}$. Thus the general representation problem for $G$ has been reduced to: (a) determining the quasi-dual $\tilde{G}$, i.e., determining the "building blocks," and (b) determining the canonical measure lattice on $\tilde{G}$, i.e., determining the "blueprints" which describe how the general representation is to be constructed from primary representations. Part (b) is a new aspect of the representation problem which is not present 
in the type I case as the canonical measure lattice is then simply the lattice of all $\sigma$-finite measure classes on $\hat{G}$. It is hoped that additional investigation will give more detailed knowledge of the canonical measure lattice for the nontype I case, than is given in the somewhat superficial characterization (Proposition 7) presented in this paper.

We remark at this point that although the previous statements are given in terms of group representations, completely analogous results hold for bounded *-representations of separable Banach *-algebras, i.e., for bounded *-algebra homomorphisms into bounded linear operators on a separable Hilbert space. Indeed, the theory will be formulated so as to apply both to unitary representations of groups and to *-representations of algebras.

In $\$ 1$ we formulate our problem, pin down our basic definitions, and describe the object, $Q$, which we plan to characterize. In $\$ 2$ we define and describe our new dual-object, the quasi-dual. In $\$ 3$ we obtain some properties of the central decomposition which we will need in the sections to follow. In $\$ 4$ we connect the two previous sections by showing that the quasi-dual may always be used as the Borel space in the central decomposition. We also show in this section that two representations are quasi-equivalent if and only if the corresponding components in their central decompositions are quasiequivalent. In $\$ 5$ we examine the lattice of measure classes determined on the quasi-dual by means of the decomposition theory developed in $\$ 4$. In particular we study the correspondence thus generated between the elements of $Q$ and measure classes on the quasi-dual. In $\$ 6$ we develop a formulation of multiplicity theory which (a) meshes in a natural manner with the characterization of representations up to quasi-equivalence developed in the previous sections, (b) distinguishes representations, up to unitary equivalence, within a quasi-equivalence class, and (c) casts the multiplicity theory for type I, II and III representations into the same mold. $\$ 7$ outlines the few modifications needed to make the entire theory applicable to projective representations.

I wish to thank Professors Feldman, Fell, Glimm and Mackey for some helpful conversations relating to the subject matter of this paper.

Professor J. Dixmier, after reading the first draft of this work, communicated to the author by letter the important result (Theorem 1) that the subset of all primary representations $G^{p}$ is a Borel subset of the set of all concrete representations $G^{c}$. (Cf. §2.) Lemmas 2 and 3, and Theorem 1, including the proofs, are the work of Professor Dixmier and we appreciate his willingness to have them incorporated into this paper. Theorem 1 has improved many of the subsequent results and in some places has greatly simplified the proofs. The reader may wish to compare this presentation with the formulation of the theory [4] developed before Professor Dixmier made his contribution.

1. Formulation of the problem. Throughout this paper W will denote a fixed separable locally compact group or a fixed separable Banach *-algebra. When $W$ is a group, the term "representation" shall mean a homomorphism 
$x \rightarrow L_{x}$ of $w$ into the group of all unitary mappings of some separable Hilbert space, $\mathfrak{F}(L)$, onto itself such that $x \rightarrow L_{x}(\phi)$ is a continuous function from $W$ to $\mathfrak{F C}(L)$ for all $\phi$ in $\mathfrak{F}(L)$. When $W$ is a Banach *-algebra, the term "representation" shall mean a homomorphism $x \rightarrow L_{x}$ of $w$ into the algebra of all bounded linear operators on some separable Hilbert space $\mathfrak{F}(L)$ such that $\left(L_{x}\right)^{*}=L_{x} *$ for all $x \in W$, and $\left\|L_{x}\right\| \leqq\|x\|$ for all $x \in W$. Two representations $L$ and $M$ of $w$ are said to be equivalent and we write $L \simeq M$ if there exists a unitary mapping $U$ of $\mathfrak{F}(L)$ onto $\mathfrak{F}(M)$ such that $U L_{x} U^{-1}=M_{x}$ for all $x$ in W. Let $R(L, L)$ denote the ring of all bounded linear operators on $\mathfrak{H C}(L)$, which commute with every element of the range of $L . L$ is said to be irreducible if the vector space dimension of $R(L, L)$ is one.

Given a group $G$, one can construct its group algebra $a_{G}$, the set of all equivalence classes of complex valued functions on $G$ which are integrable with respect to left invariant Haar measure. Multiplication is defined by convolution, i.e., $[f * g](x)=\int_{G} f(x y) g\left(y^{-1}\right) d y$. We define an involution in $a_{G}$ by $f^{*}(x)=f\left(x^{-1}\right) \Delta\left(x^{-1}\right)$ where $\Delta(x)$ is a continuous homomorphism of $G$ into the real numbers satisfying $\int_{G} f(x t) d x=\int_{G} \Delta(t) f(x) d x$ for all $f \in Q_{G}$ and $t \in G$. (Cf. [11, p. 120; and 15, p. 154].) Furthermore there is a one-to-one correspondence between the set of all representations $T$ of $G$ and the set of all representations $T^{\prime}$ of $Q_{G}$ which are nowhere trivial (sometimes called proper) in the sense that the linear union of the ranges of the $T_{f}^{\prime}$ for $f \in a_{G}$, is dense in $\mathfrak{F C}\left(T^{\prime}\right)$. The correspondence between $T$ and $T^{\prime}$ is defined by the requirement that $\left(T_{f}^{\prime} \phi, \psi\right)=\int_{G} f(s)\left(T_{\bullet} \phi, \psi\right) d s$ for all $f \in Q_{G}$ and $\phi, \psi \in \mathcal{H C}(T)$.

LEMMA 1. Let $T$ be any unitary representation of a separable locally compact group $G$ and let $T^{\prime}$ denote the corresponding nowhere trivial *-representation of its group algebra $\mathfrak{Q}_{G}$. Then $\mathfrak{R}(T, T)=\mathbb{R}\left(T^{\prime}, T^{\prime}\right)$.

Proof. Left to the reader.

From Lemma 1 it follows that the von Neumann algebra $a(T)$ generated by the range of the representation $T$ of the group $G$ is identical with $Q\left(T^{\prime}\right)$, the von Neumann algebra generated by the range of the corresponding *-representation $T^{\prime}$ of the group algebra $\mathfrak{Q}_{G}$. Thus we have that $T$ is irreducible if and only if $T^{\prime}$ is irreducible. $T$ is primary if and only if $T^{\prime}$ is primary. $T$ and $T^{\prime}$ are of the same von Neumann type. The concept of "group $C^{*}$ algebra" has been introduced in [5, p. 369]. There is an exactly similar correspondence between representations of the group and *-representations of its "group $C^{*}$-algebra." Lemma 1 and the statements made above also apply to this correspondence. For the most part, it is these properties of the correspondence between representations of a group and of its group algebra (or of its group $C^{*}$-algebra) which enables one to develop a decomposition theory for representations of groups simultaneously with a decomposition theory for *-representations of Banach *-algebras.

The object of investigation is the collection $Q$ of all quasi-equivalence classes of representations of $w . Q$ is partially ordered by the covering relation 
defined above. In discussing this ordering it is convenient to adjoin an element, denoted 0 , to $Q$. (One might take 0 to be the empty set.) We extend the partial ordering by defining $L\} 0$ for every $L \in Q$. Then two points $L, M \in Q$, are disjoint if and only if the infimum of $L$ and $M, L \wedge M$, is equal to 0 . We shall continue to use the notation $Q$ for the original collection with this distinguished point adjoined.

Proposition 1. $Q$ is a distributive $\sigma$-lattice in which one may take relative complements. (By the "relative complement," $L-M$, where $L, M \in Q$, we mean an element $N \in Q$ such that $N$ \& $M$ and $N \bigvee M=L \bigvee M$.)

Proof. Since there is a one-to-one order preserving correspondence between the $Q$ associated with a group $G$ and that associated with its group algebra $a_{G}$, it is sufficient to verify the statement for the case where $W$ is a Banach *-algebra.

Given a countable collection of elements of $Q$, one need only choose one representative of each class in the given collection, form their direct sum in the ordinary representation theoretic manner, and then take the quasiequivalence class containing the representation constructed in this way. It is easy to verify that the element of $Q$ determined in this manner is the supremum of the given countable collection of elements of $Q$. Hence $Q$ is $\sigma$-complete.

Let $L, M \in Q$. We next show that there exists an element $N \in Q$ such that $N=L-M$. Let $\bar{L}$ and $\bar{M}$ denote representatives of the classes $L$ and $M$ respectively. By [12, Theorem 1.11], $\bar{L}$ may be written uniquely as the direct sum of two representations, say $\bar{L}=\bar{L}_{1}+\bar{N}$ such that $\bar{L}_{1}\{\bar{M}$ and $\bar{N} \circ \bar{M}$. Let $L_{1}$ and $N$ denote the quasi-equivalence class containing $\bar{L}_{1}$ and $\bar{N}$ respectively. Then $N \circ M$ and further, $L \bigvee M=\left(L_{1} \bigvee N\right) \bigvee M=\left(L_{1} \bigvee M\right) \bigvee(N \bigvee M)$. However since $M\} L_{1}$ we have $L_{1} \bigvee M=M$. Thus $L \bigvee M=M \bigvee(N \vee M)=N \vee M$. Thus $N=L-M$.

Given a triplet of elements, $L, M$ and $N$ in $Q$, the reader may easily verify the identities:

$$
L \wedge M=(L \vee M)-[(L-M) \vee(M-L)]
$$

and

$$
L-(M \vee N)=(L-M) \wedge(L-N) .
$$

These identities may in turn be used to verify that the lattice is distributive.

On the basis of Proposition 1, it is natural to look for a characterization of $Q$ by means of a lattice ideal of measure classes on some Borel space. Our next step then is to determine this Borel space, which will be our "dual object."

2. The quasi-dual. Let $\tilde{\mathcal{W}}$ denote the collection of all primary classes in $Q$, the lattice of quasi-equivalence classes of representations of $W$. The purpose of this section is to introduce a Borel structure in $\tilde{\mathbb{W}}$. That is, we wish to 
specify a $\sigma$-ring of subsets of $\tilde{\mathscr{W}}$ which we will call the Borel sets of $\tilde{\mathcal{W}}$. In the author's first formulation of this theory (cf. [4]) a somewhat contrived Borel structure was specified in $\tilde{\mathcal{W}}$. Professor Jacques Dixmier has since communicated to the author certain results which enables one to use a far more natural Borel structure in $\tilde{\mathbb{W}}$.

For each $n=1,2,3, \cdots$, let $\mathfrak{H}_{n}$ denote the $n$ dimensional Hilbert space of $n$-tuples of complex numbers with the usual inner product. Let $\mathfrak{H}_{\infty}$ denote the classical Hilbert space of finite sequences $\left\{c_{k}\right\}$ of complex numbers such that $\sum_{k=1}^{\infty}\left|c_{k}\right|^{2}<\infty$, with the usual inner product. Let $w^{c}$ denote the set of all representations $L$ of $W$ such that the representation space $\mathcal{H C}(L)$ of $L$ is one of the spaces $\mathfrak{H C}_{\infty}, \mathfrak{H}_{1}, \mathfrak{H C}_{2}, \cdots$. (In the case where $\mathfrak{W}$ is a Banach *-algebra, $W^{c}$ is the set of all nowhere trivial *-representations with representation space one of the $\mathfrak{H}_{n}$.) We do not identify equivalent representations. We give $W^{c}$ the Mackey-Borel structure [15, p. 149]. That is, we denote by $W_{n}^{c}$ the subset of $\mathscr{W}^{c}$ consisting of all representations $L$ such that $\mathfrak{F}(L)=\mathfrak{H}_{n}$, and give $W^{c}$ the smallest Borel structure having the properties that each $W_{n}^{c}$ is a Borel set and, for each $n$, for each $\phi, \psi \in \mathcal{F}_{n}$, and for each $x \in W$, the function $L \rightarrow\left(L_{x} \phi, \psi\right)$ is a Borel function on $W_{n}^{c}$. Then $W^{c}$ is a standard Borel space [15, Theorem 8.1]. (A Borel space is called standard if it is Borel isomorphic to the Borel space associated with a complete separable metric space.) Let $w^{p}$ denote the subset of $w^{c}$ consisting of all primary representations. The following two lemmas will enable us to prove the important fact that $W^{p}$ is a Borel subset of $W^{c}$. Let $\mathfrak{F}$ be a separable Hilbert space and let $Z$ be the set of representations of $W$ with representation space $\mathcal{H}$. Give $Z$ the smallest topology such that $L \rightarrow\left(L_{x} \phi, \psi\right)$ is a continuous function for all $x \in W$ and $\phi, \psi \in \mathfrak{H C}$. Let $L(\mathcal{H C})$ denote the set of bounded linear operators on $\mathfrak{H C}$ and let $L_{n}(\mathfrak{F C})$ denote the closed ball of radius $n$ in $L(\mathcal{H C})$. Note that for fixed $x \in W$, the map $L \rightarrow L_{x}$ is a continuous map of $Z$ into $L(\mathcal{F C})$, where $L(\mathcal{H C})$ has the strong operator topology. Indeed if we have a sequence $\left\{L^{i}\right\}$ which converges to $L$ in $Z$, then $L_{x}^{i} \rightarrow L_{x}$ in the weak operator topology and thus it is sufficient to show that $\left\|L_{x}^{i} \phi\right\| \rightarrow\left\|L_{x} \phi\right\|$ for all $\phi \in \mathcal{F}$. This condition is obviously satisfied when $W$ is a group, as all the operators are then unitary. If W is a *-algebra, then $\left\|L_{x}^{i} \phi\right\|^{2}=\left(L_{x}^{i} \phi, L_{x}^{i} \phi\right)=\left(L_{x}^{i}{ }_{x} \phi, \phi\right) \rightarrow\left(L_{x}{ }^{*} \phi, \phi\right)=\left(L_{x} \phi, L_{x} \phi\right)$ $=\left\|L_{x} \phi\right\|^{2}$, for all $\phi \in \mathcal{H C}$.

LEMMA $2\left({ }^{2}\right)$. Let $d$ be a metric on $L_{1}(3 \mathrm{C})$ compatible with the weak topology, $S$ an element of $L_{1}(\mathcal{H C}), a$ and $b$ non-negative real numbers, and $x_{1}, x_{2}, \cdots, x_{n}$ elements of $w$. Let $Y$ denote the set of all $L \in Z$ having the following property: There exists $T_{1}, T_{2}, \cdots, T_{n}$ in $L_{b}(\mathfrak{H C}) \cap R(L, L)$ such that $L_{x_{1}} T_{1}+\cdots+L_{x_{n}} T_{n}$ $\in L_{1}(\mathfrak{H C})$ and $d\left(L_{x_{1}} T_{1}+\cdots+L_{x_{n}} T_{n}, S\right) \leqq a$. Then $Y$ is closed in $Z$.

Proof. Let $\left\{L^{i}\right\}$ denote a family of elements of $Y$, indexed by a directed system, and let $L$ denote an element of $Z$ such that $L^{i} \rightarrow L$. Then for each $i$,

(2) This result was obtained by J. Dixmier. 
there exists $T_{1}^{t}, \cdots, T_{n}^{i}$ in $L_{b}(\mathfrak{H C}) \cap R\left(L^{i}, L^{i}\right)$ such that $L_{x_{1}}^{i} T_{1}^{i}+\cdots+L_{x_{n}}^{i} T_{n}^{t}$ is contained in $L_{1}(\mathfrak{F C})$ and $d\left(L_{x_{1}}^{i} T_{1}^{i}+\cdots+L_{x_{n}}^{i} T_{n}^{i}, S\right) \leqq a$. Since $L_{b}(\mathfrak{F C})$ is weakly compact one may, by taking a subnet of $\left\{L^{i}\right\}$, assume that the $T_{1}^{q}, \cdots, T_{n}^{i}$ converge weakly to some elements $T_{1}, \cdots, T_{n}$ in $L_{b}(\mathcal{F C})$. Suppose $x \in W$ and $\phi, \psi \in \mathcal{F}$. Then we have that $\left(T_{1}^{i} L_{x}^{i} \phi, \psi\right)=\left(L_{x}^{i} \phi, T_{1}^{* *} \psi\right)$ $\rightarrow\left(L_{x} \phi, T_{1}^{*} \psi\right)=\left(T_{1} L_{x} \phi, \psi\right)$ and

$$
\left(L_{x}^{i} T_{1}^{i} \phi, \psi\right)=\left(T_{1}^{i} \phi, L_{x}^{i *} \psi\right) \rightarrow\left(T_{1} \phi, L_{x}^{*} \psi\right)=\left(L_{x} T_{1} \phi, \psi\right) .
$$

Hence $\left(T_{1} L_{x} \phi, \psi\right)=\left(L_{x} T_{1} \phi, \psi\right)$. Therefore $T_{1} \in R(L, L)$ and, similarly, $T_{2}, \cdots, T_{n} \in \mathbb{R}(L, L)$. Furthermore, we have $\left(\left(L_{x_{1}}^{i} T_{1}^{i}+\cdots+L_{x_{n}}^{i} T_{n}^{i}\right) \phi, \psi\right)$ $\rightarrow\left(\left(L_{x_{1}} T_{1}+\cdots+L_{x_{n}} T_{n}\right) \phi, \psi\right)$ and hence $L_{x_{1}}^{i} T_{1}^{i}+\cdots+L_{x_{n}}^{i} T_{n}^{i}$ converges weakly to $L_{x_{1}} T_{1}+\cdots+L_{x_{n}} T_{n}$. Thus it follows that $L_{x_{1}} T_{1}+\cdots+L_{x_{n}} T_{n}$ $\in L_{1}(\mathfrak{F C})$ and $d\left(L_{x_{1}} T_{1}+\cdots+L_{x_{n}} T_{n}, S\right) \leqq a$. Thus $L \in Y$.

Lemma $3\left({ }^{2}\right)$. Let $d$ be a metric in $L_{1}(\mathfrak{H C})$ compatible with the weak topology, $\left(x_{1}, x_{2}, \cdots\right)$ a sequence everywhere dense in $w$ and $\left(S_{1}, S_{2}, \cdots\right)$ a sequence weakly everywhere dense in $L_{1}(\mathfrak{H C})$. Let $k, n, p$ denote positive integers. Let $Z_{k, n, p}$ denote the set of all $L \in Z$ possessing the following property: There exists $T_{1}, T_{2}, \cdots, T_{n}$ in $L_{n}(\mathfrak{F}) \cap R(L, L)$ such that $L_{x_{1}} T_{1}+\cdots+L_{x_{n}} T_{n} \in L_{1}(\mathfrak{F C})$ and $d\left(L_{x_{1}} T_{1}+\cdots+L_{x_{n}} T_{n}, S_{k}\right) \leqq 1 / p$. Then $Z_{k, n, p}$ is closed in $Z$ and $Y=\bigcap_{k, p} \bigcup_{n} Z_{k, n, p}$ is the set of those $L \in Z$ which are primary.

Proof. Lemma 2 implies that each $Z_{k, n, p}$ is closed.

For all $L \in Z$, let $\mathfrak{x}(L)$ denote the von Neumann algebra generated by $\mathfrak{Q}(L) \cup \mathfrak{R}(L, L)$. Then $L$ is primary if and only if $\mathfrak{T}(L)=L(\mathfrak{C})$.

Suppose first that $L \in Y$. We will show for each $k$ that $S_{k} \in \mathfrak{T}(L)$ and thus that $\mathfrak{T}(L)=L(\mathfrak{H})$. Let $p$ denote a positive integer. Then there exists a positive integer $n$ such that $L \in Z_{k, n, p}$. Thus there exists $T_{1}, \cdots, T_{n} \in L_{n}(\mathfrak{F C})$ $\cap \Re(L, L)$ such that $L_{x_{1}} T_{1}+\cdots+L_{x_{n}} T_{n} \in L_{1}(\mathfrak{F C})$ and

$$
d\left(L_{x_{1}} T_{1}+\cdots+L_{x_{n}} T_{n}, S_{k}\right) \leqq 1 / p .
$$

Since $L_{x_{1}} T_{1}+\cdots+L_{x_{n}} T_{n} \in \mathfrak{x}(L)$ we have $d\left(\mathfrak{T}(L) \cap L_{1}(\mathfrak{H C}), S_{k}\right) \leqq 1 / p$. Since $p$ was arbitrary we have proved that $S_{k} \in \mathfrak{N}(L)$. Hence $L$ is primary.

Conversely, suppose that $L \in Z$ and $L$ is primary, i.e., suppose $\Re(L)$ $=L(\mathfrak{C})$. Let $k$ and $p$ be two positive integers. We will complete the proof of the lemma by showing that there exists an $n$ such that $L \in Z_{k, n, p}$. Let $C(L)$ denote the space of finite sums of operators of the form $L_{x} T$ where $x \in W$ and $T \in R(L, L)$. Then $C(L)$ is a *algebra of operators whose weak closure is $\Re(L)=L(\Im)$. According to the Kaplansky density theorem (cf. [1, p. 46]) there exists an operator $P \in C(L)$ such that $\|P\|<1$ and $d\left(P, S_{k}\right)<1 / p . P$ is of the form $P=L_{y_{1}} T_{1}+\cdots+L_{y_{m}} T_{m}$ where $y_{1}, \cdots, y_{m} \in W$ and $T_{i} \in R(L, L)$. By choosing, for each $j$, an element $x_{i j}$ of the dense sequence $\left\{x_{i}\right\}$ sufficiently close to $y_{j}, j=1, \cdots, m$, we will have $\left\|L_{x_{i_{1}}} T_{1}+\cdots+L_{x_{i_{m}}} T_{m}\right\| \leqq 1$ and 
$d\left(L_{x_{i_{1}}} T_{1}+\cdots+L_{x_{i_{m}}} T_{m}, S_{k}\right) \leqq 1 / p$. Hence if one chooses $n$ to be any integer greater than $i_{1}, \cdots, i_{m}$ and $\left\|T_{1}\right\|, \cdots,\left\|T_{m}\right\|$, we will have $L \in Z_{k, n, p}$.

TheOREM $1\left(^{2}\right)$. The set $w^{p}$ is a Borel subset of $w^{c}$.

Proof. Give each $W_{n}^{c}$ the smallest topology for which all the functions $L \rightarrow\left(L_{x}, \phi, \psi\right)$ are continuous, for all $x \in W$ and $\phi, \psi \in \mathcal{H}_{n}$. Then the Borel structure in $\mathscr{W}_{n}^{c}$ defined above is the smallest Borel structure containing this topology. Hence by Lemma 3, the primary representations in $W_{n}^{c}$ form a Borel subset of $W_{n}^{c}$, for each $n=\infty, 1,2, \cdots$. Hence $W^{p}$ is a Borel subset of $W^{c}$.

COROLlaRY. The set $W^{p}$, considered as a Borel subspace of $W^{c}$, is a standard Borel space.

Proof. The corollary follows immediately from the above theorem and [15, Corollary 1 to Theorem 3.2, and Theorem 8.1].

Imitating the method Professor Mackey has used in defining a Borel structure in $\hat{\mathscr{W}}$ (cf. [15]) we give $\tilde{\mathscr{W}}$ the Borel structure determined as a quotient of $\mathscr{W}^{p}$. That is to say, we give $\tilde{\mathscr{W}}$ the largest Borel structure such that the canonical mapping of $W^{p}$ into $\tilde{W}$ is Borel.

In the next proposition we examine the relationship between the Borel structure of $W^{p}$ and the quasi-equivalence relation. If $S$ is a subset of $w^{p}$, let $S^{q}$ denote the set of elements of $w^{p}$ which are quasi-equivalent to some element of $S$.

Proposition 2. If $B$ is a Borel subset of $w^{p}$, then $B^{q}$ is analytic and hence measurable. Further if $B$ intersects each quasi-equivalence class in at most one point, then $B^{a}$ is Borel.

Proof. By the corollary to Theorem 1 and the definition of standard, there exists a metric for ${ }^{w^{p}}$ which will generate the Borel structure of ${ }^{{ }^{p}}$ and with respect to which $W^{p}$ is a complete separable metric space. It is this fact which enables us to use the results of [10] in what follows. Form the topological Cartesian product $W^{p} \times W^{p}$. Then the projections of $w^{p} \times W^{p}$ into $W^{p}$ are continuous and thus Borel. Theorem 8.2 of [15] states that $g(L, M)$ is a Borel function on $W^{p} \times W^{p}$. $(\mathcal{G}(L, M)$ is the vector-space dimension of the set of all bounded linear transformations $T$ from $\mathfrak{H C}(L)$ to $\mathfrak{H C}(M)$ such that $T L_{x}=M_{x} T$ for all $x \in W$.) Thus $S=\{(L, M): \mathscr{g}(L, M) \neq 0\} \cap\left(W^{p} \times B\right)$ $=\left\{(L, M): L \sim M, L \in W^{p}, M \in B\right\}$ is a Borel subset of $w^{p} \times W^{p}$. By $[10$, p. 366] $B^{q}$, which is the projection of $S$ into $w^{p}$, is analytic and thus, by $[10$, p. 391], measurable.

If $B$ intersects each quasi-equivalence class in at most one point, then the projection of $S$ into $W^{p}$ is one-to-one. Hence by [15, Theorem 3.2] the range of this projection, namely $B^{q}$, is Borel.

Corollary. $\tilde{\mathcal{W}}$ is a separated Borel space. Indeed, every point of $\tilde{\mathcal{W}}$ is a Borel set. 
Proof. If $\tilde{L}$ is a point of $\tilde{\mathscr{W}}$, choose $L \in W^{p}$ such that $L \in \tilde{L} .\{L\}$ is a Borel set in $\mathscr{W}^{p}$ and hence by Proposition $2,\{L\}^{q}$ is Borel. Thus $\{\tilde{L}\}$ is a Borel set in $\tilde{\mathbb{W}}$.

3. The central decomposition. We have described our dual object $\tilde{\mathscr{W}}$ and we must now develop a method of associating a measure $\mu$ on $\tilde{W}$ with each representation $L$ of $W$. This will be effected by the central decomposition of $L$ (sometimes called the "canonical decomposition"). This section will be devoted to the description of this concept. (Cf. [14;15;17;19 and 20].)

Recall that a Borel space $S$ is called standard if it is Borel isomorphic with the Borel space associated with a complete separable metric space. A measure $\mu$ on a Borel space $S$ is called standard if $S$ contains a Borel set $B$ such that $\mu(S-B)=0$ and $B$ is a standard Borel space. For completeness we give the definition of direct integral representation as formulated by $\mathrm{G}$. W. Mackey in [15]. Let $\mu$ denote a $\sigma$-finite standard measure in the Borel space $S$ and suppose $y \rightarrow L^{\nu}$ is a $\mu$-measurable mapping of $S$ into $w^{c}$. For each $n=\infty, 1,2, \cdots$, let $\mathfrak{H}_{n}$ denote the classical Hilbert space of $n$-tuples of complex numbers referred to in the definition of $w^{c}$ above. Let $S_{n}$ denote the subset of $S$ consisting of those $y \in S$ for which the representation space of $L^{y}, \mathfrak{F C}\left(L^{y}\right)$, is $\mathcal{F C}_{n}$. Form the Hilbert space $\mathfrak{F C}(M)$ consisting of all functions $f$ from $S$ to $\bigcup_{n=\infty, 1,2} \ldots \mathcal{K}_{n}$ such that (a) $f(y) \in \mathcal{K}_{n}$ for all $y \in S_{n}$; (b) for each $n=\infty, 1,2, \cdots,(f(y), \phi)$ is a Borel function on $S_{n}$, for all $\phi \in \mathfrak{F}_{n}$; (c) $\int_{S}(f(y), f(y)) d \mu(y)<\infty$. Define an inner product in $\mathcal{H C}(M)$ by setting $(f, g)=\int_{S}(f(y), g(y)) d \mu(y)$ for $f, g \in \mathfrak{F C}(M)$ and identify $f$ and $g$ if $\|f-g\|=0$. For each $x \in W$ and $f \in \mathcal{H}(M)$, let $M_{x}(f)=g$ where $g(y)=L_{x}^{y}(f(y))$ for all $y \in S$. Then the map $x \rightarrow M_{x}$ is a representation of $w$ which we define to be the direct integral of $y \rightarrow L^{y}$ and we write $M=\int_{S} L^{y} d \mu(y)$.

To each Borel subset $B$ of $S$ we associate a projection $E(B)$ on $\mathfrak{H C}(M)$ defined by $(E(B) f)(y)=\chi(B) f(y)$ where $\chi(B)=1$ when $y \in B$ and $\chi(B)=0$ when $y \notin B$. The function $B \rightarrow E(B)$ is called a projection valued measure. The decomposition $M=\int_{S} L^{v} d \mu(y)$ is called central (or canonical) when the range of the corresponding projection valued measure is just the set of all projections contained in the center of $R(M, M)$. Every representation $L$ of $W$ has a central decomposition. Further almost all the components of the central decomposition are primary [14, Theorem 2.5]. The central decomposition has the characterizing property that any decomposition of a representation $L$ into primary representations over a standard Borel space is necessarily a "refinement," in the obvious sense, of the central decomposition of $L$. Further the central decomposition of a representation is unique in the sense that if $L \simeq \int_{S^{\prime}} L^{y^{\prime}} d \mu^{\prime}\left(y^{\prime}\right)$ is another central decomposition for $L$, then there exists a measure space isomorphism between $(S, \mu)$ and $\left(S^{\prime}, \mu^{\prime}\right)$, say $y \rightarrow y^{\prime}$ such that $L^{y} \simeq L^{y^{\prime}}$ for $\mu$-almost all $y$ in $S$.

A. Guichardet [8] has proved that if a multiplicity-free representation is decomposed into irreducible representations, then the components are, almost everywhere, two-by-two disjoint. A perturbation of his proof gives 
the following more general result (Proposition 3). We need a proof directly applicable to groups in order to obtain an easy generalization to projective representations, which will be discussed in $\$ 7$. For this reason we present the revised proof for the case where $W$ is a separable locally compact group. The required revision of Guichardet's proof in the case where $W$ is a *algebra is left to the interested reader.

Proposition 3. Let $L$ denote a representation of W with a decomposition $L \simeq \int_{S} L^{y} d \mu(y)$ over a separable Borel space $S$, such that the range $B$ of the corresponding projection-valued measure is contained in the center, $\mathcal{C} R(L)$, of the commuting algebra $R(L, L)$. Then there exists a Borel subset $S^{\prime}$ of $S$ such that $\mu\left(S-S^{\prime}\right)=0$ and $L^{y} \delta L^{y^{\prime}}$ whenever $y, y^{\prime} \in S^{\prime}$ and $y \neq y^{\prime}$.

Proof. We consider the case where $W$ is a separable locally compact group. Let $\mathfrak{A}$ denote the *-algebra of operators consisting of all complex linear combinations of elements $L_{x}$ where $x \in \mathfrak{W}$. Since $\mathfrak{A}$ contains the identity operator we have $[1$, p. 44$]$ that the strong closure of $\mathfrak{A}$ is equal to the weak closure of $\mathfrak{A}$, which is just the von Neumann algebra $a(L)$ generated by the set $\left\{L_{x}: x \in W\right\}$. Thus if $T \in Q(L)$, then $T$ is the strong limit of a sequence $T_{i}$ such that $T_{i}=\sum_{j} a_{i j} L_{x_{i j}}$ where the $a_{i j}$ are complex numbers and $x_{i j} \in W$. Since $B \subset \mathcal{C} R(L)$, every $T \in Q(L)$ commutes with every element of $B$ and is therefore decomposable. Let $T=\int_{S} T^{y} d \mu(y)$ and $T_{i}=\int_{S}\left(\sum_{j} a_{i j} L_{x_{i j}}^{\nu}\right) d \mu(y)$ $=\int_{S} T_{i}^{y} d \mu(y)$. By [1, Proposition 4, p. 162] we may assume that $T_{i}^{y}$ converges strongly to $T^{y}$ for all $y \in S(T)$ where $S(T)$ is a Borel subset of $S$ such that $\mu(S-S(T))=0$. Now suppose $y$ and $y^{\prime}$ are two points of $S(T)$ such that $L^{y}$ and $L^{y^{\prime}}$ are not disjoint. Then $R\left(L^{y}, L^{y^{\prime}}\right)$ contains a nonzero element, say $R\left(y, y^{\prime}\right)$. Thus for all $i, T_{i}^{y^{\prime}} R\left(y, y^{\prime}\right)=R\left(y, y^{\prime}\right) T_{i}^{y}$ and hence, by $[1$, p. 32], $T^{\nu^{\prime}} R\left(y, y^{\prime}\right)=R\left(y, y^{\prime}\right) T^{\nu}$.

Let $P$ denote the projection valued measure on $S$ associated with the decomposition of $L$ and let $\left\{E_{i}\right\}$ denote a countable separating family of Borel subsets of $S$. Then each $P_{E_{n}} \in B$ and $B \subset \mathcal{C} R(L) \subset a(L)$ and thus we may apply our previous considerations to each $P_{E_{n}}$. Thus for each $n$ there exists a Borel subset $S_{n}$ of $S$ such that $\mu\left(S-S_{n}\right)=0$ and if $L^{y}$ and $L^{y^{\prime}}$ are not disjoint for $y, y^{\prime} \in S_{n}$, then there exists a nonzero $R\left(y, y^{\prime}\right) \in R\left(L^{y}, L^{y^{\prime}}\right)$ such that $P_{E_{n}}^{v^{\prime}} R\left(y, y^{\prime}\right)=R\left(y, y^{\prime}\right) P_{E_{n}}^{v}$. However each $P_{, E_{n}}^{v}$ is either 0 or the identity operator. Hence the last equality implies that $P_{E_{n}}^{v^{\prime}}=P_{E_{n}}^{v}$. Let $S^{\prime}=\bigcap_{n=1,2, \ldots} \ldots S_{n}$. Then $\mu\left(S-S^{\prime}\right)=\sum_{n} \mu\left(S-S_{n}\right)=0$. Now suppose $y, y^{\prime} \in S^{\prime}$ and that $L^{y}$ and $L^{y^{\prime}}$ are not disjoint. Then $P_{E_{n}}^{v^{\prime}}=P_{E_{n}}^{v}$ for $n=1,2, \cdots$, which implies that $y^{\prime} \in E_{n}$ if and only if $y \in E_{n}$. Since the collection $\left\{E_{n}\right\}$ separates points we have $y=y^{\prime}$.

COROLlARY. The central decomposition of a representation has the property that, after eliminating a set of measure zero, the components are two-by-two disjoint primary representations $\left({ }^{3}\right)$.

(3) This result has been announced by M. A. Naimark [18, Theorem 1]. 
4. Decomposition theory. In this section we connect the two previous sections by proving that the Borel space $S$ associated with the central decomposition of a representation of $W$ may always be taken to be $\tilde{\mathscr{W}}$. We then consider the correspondence thus induced between representations of $w$ and measures on $\tilde{\mathcal{W}}$.

THEOREM 2. The central decomposition of a representation of $W$ may always be taken over the space $\tilde{W}$. More explicitly, to every representation $L$ of $W$ there corresponds a standard $\sigma$-finite Borel measure $\mu$ on $\tilde{\mathcal{W}}$ and a $\mu$-measurable map $y \rightarrow L^{y}$ of $\tilde{W}$ into $w^{c}$ such that $L^{y} \in y, L \simeq \int \tilde{W} L^{y} d \mu(y)$, and the range of the projection valued measure associated with this decomposition is the set of all projections in $\mathbb{C} R(L)$.

Proof. Let $L \simeq \int_{S} L^{v} d \mu(y)$ be the central decomposition of $L$ over some standard Borel space $S$, where $y \rightarrow L^{y}$ is a Borel map of $S$ into $W^{c}$. By the corollary to Proposition 3 we may assume that the components $L^{y}$ are twoby-two disjoint primary representations. Thus the map $y \rightarrow L^{y}$ is a one-to-one Borel map of $S$ into $W^{p}$, which, by $[15$, Theorem 3.2] is a Borel isomorphism of $S$ onto a Borel subset $S^{\prime}$ of $w^{p}$. Let $\varphi$ denote the canonical mapping of $S^{\prime}$ into $\tilde{\mathscr{W}}$ defined by sending a representation into the quasi-equivalence class containing it. Then $\varphi$ is a one-to-one mapping since the elements of $S^{\prime}$ are two by-two disjoint. Further the second part of Proposition 2 implies that $S^{\prime \prime}=\varphi\left(S^{\prime}\right)$ is a Borel subset of $\tilde{\mathcal{W}}$ and that $\varphi^{-1}$ is a Borel map of $S^{\prime \prime}$ onto $S^{\prime}$. If $B$ is a Borel subset of $S^{\prime \prime}$, then $\bar{B}=\left\{L: L \in W^{p}\right.$ and $L \in y$ for some $\left.y \in B\right\}$ is a Borel subset of $w^{p}$ by the definition of Borel structure in $\tilde{W}$. Hence $\bar{B} \cap S^{\prime}=\varphi^{-1}(B)$ is a Borel subset of $S^{\prime}$. Thus $\varphi$ is a Borel map and hence a Borel isomorphism of $S^{\prime}$ onto $S^{\prime \prime}$. Thus $S$ and $S^{\prime \prime}$ are Borel isomorphic and the rest of the theorem follows at once.

It is well known that in any direct integral decomposition of a representation, the measure is determined only up to equivalence. Thus the standard measure $\mu$ on $\tilde{W}$ given by Theorem 2 is not unique, although the ring of $\mu$ null sets is determined uniquely. Hence the statement of Theorem 2 may be refined as follows.

CoRollary. To each representation $L$ of $W$ there corresponds a unique standard measure class $\mu$ on $\tilde{\mathbb{W}}$, a Borel set $B$ such that $\tilde{\mathbb{W}}-B$ is a $\mu$-null set and $B$ is a standard Borel space, and a Borel map $y \rightarrow L^{y}$ of $B$ into wc such that $L^{\nu} \in y$ for all $y \in B$ and $\int_{B} L^{y} d \mu(y)$ is the central decomposition of $L$.

The remainder of this section will be devoted to showing that there exists a one-to-one correspondence between elements of $Q$ (i.e., quasi-equivalence classes of representations) and certain standard measure classes on $\tilde{\mathscr{W}}$. We do this by proving that two representations of $W$ are quasi-equivalent if and only if they correspond, according to the corollary to Theorem 2, to the same standard measure class on $\tilde{\mathscr{W}}$. In order to obtain this result, however, we must first digress to clarify the relationship between the decomposition 
theory of representations and the well-known decomposition theory for von Neumann algebras.

Suppose $L \simeq \int_{S} L^{y} d \mu(y)$ is a direct integral decomposition (not necessarily central) of a representation $L$ of W. Let $Q(L)$ and $Q\left(L^{y}\right)$ denote the von Neumann algebras generated by the range of $L$ and the range of $L^{y}$ respectively. Then it is always true that $y \rightarrow \mathfrak{Q}\left(L^{y}\right)$ is a measurable field of von Neumann algebras and furthermore $a(L) \subset \int_{S} Q\left(L^{y}\right) d \mu(y)$. (See [1] for terminology and the theory of direct integrals of von Neumann algebras.) Indeed, by choosing a dense sequence of elements of $W$ (we are assuming throughout that $W$ is separable), say $x_{1}, x_{2}, \cdots$, we obtain a sequence of operator fields, $y \rightarrow L_{x_{1}}^{y}$, $y \rightarrow L_{x_{2}}^{y}, \cdots$, such that, for $\mu$-almost all $y,\left\{L_{x_{i}}^{y}\right\}$ generates $Q\left(L^{y}\right)$. Further $\int_{S} Q\left(L^{y}\right) d \mu(y)$ is a von Neumann algebra which contains the decomposable operators $L_{x_{i}}=\int_{S} L_{x_{i}}^{y} d \mu(y)$ for all $i$, and thus $\int_{S} Q\left(L^{y}\right) d \mu(y)$ contains $Q(L)$. Note, however, that $Q(L)$ may well be distinct from $\int_{S} Q\left(L^{y}\right) d \mu(y)$. In fact this is always the case when the Boolean algebra of projections associated with the decomposition is not contained in the center of $R(L, L)$. Indeed, suppose $E$ is in the range of the associated projection valued measure and $E \notin \mathbb{C} R(L)$, the center of $R(L, L)$. In the terminology of Dixmier [1] $E$ is a diagonalizable projection and thus [1, Theorem 1, p. 178] $E \in \int_{S} Q\left(L^{y}\right) d \mu(y)$. However, since $E \in \mathbb{R}(L, L)$ and $E \in \mathbb{C} R(L)=\mathbb{R}(L, L) \cap \mathbb{Q}(L)$, we have $E \in Q(L)$. The exact situation is described in the following statement.

Proposition 4. Suppose $L=\int_{S} L^{y} d \mu(y)$. A necessary and sufficient condition for $Q(L)$ to be equal to $\int_{S} Q\left(L^{y}\right) d \mu(y)$ is that the Boolean algebra of projections $B$, associated with the given decomposition of $L$, be contained in the center of $Q(L)$.

Proof. The necessity of the condition was given in the previous remark. Suppose now the condition holds. Letting $\left\{x_{1}, x_{2}, \cdots\right\}$ denote a countable dense collection of elements of $W$ we have $[1$, Theorem 1, p. 178] that $\int_{S} Q\left(L^{y}\right) d \mu(y)$ is generated by $\left\{\int_{S} L_{x_{i}}^{\nu} d \mu(y)=L_{x_{i}}: i=1,2, \cdots\right\}$ and the collection of diagonalizable operators. But the collection of diagonalizable operators is just the von Neumann algebra generated by $B$, which is contained in $Q(L)$. Thus $\int_{S} Q\left(L^{y}\right) d \mu(y) \subset Q(L)$. $Q(L)$, as we remarked above, is always contained in $\int_{S} Q\left(L^{y}\right) d \mu(y)$.

Corollary 1. If $L$ is a representation of W with central decomposition

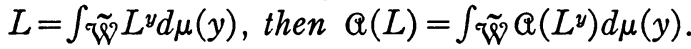

CoROllary 2. If $L$ is a representation of $W$ with a decomposition $L=\int_{S} L^{y} d \mu(y)$ such that the associated Boolean algebra of projections is contained in the center of $Q(L)$, then

$$
\Re(L, L)=\int_{S} \mathfrak{R}\left(L^{y}, L^{y}\right) d \mu(y)
$$

and 


$$
\mathfrak{C} R(L)=\int_{S} \mathfrak{e} R\left(L^{y}\right) d \mu(y) .
$$

In particular these properties hold for the central decomposition.

Proof. By Proposition 4 we have $Q(L)=\int_{S} Q\left(L^{y}\right) d \mu(y)$. Then by $[1$, Theorem 4, p. 184] we have $R(L, L)=Q(L)^{\prime}=\int_{S} Q\left(L^{y}\right)^{\prime} d \mu(y)$

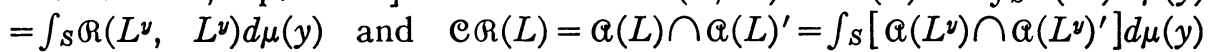
$=\int_{S} \mathbb{e} R\left(L^{\nu}\right) d \mu(y)$.

The following lemma is designed to further clarify the relation between representations and the von Neumann algebras generated by their range.

Lemma 4. If $L$ and $M$ are representations of $w$, then $L$ and $M$ are quasiequivalent if and only if there exists an algebraic isomorphism $\varphi$ of $a(L)$ onto $Q(M)$ such that $\varphi\left(L_{x}\right)=M_{x}$ for all $x \in W$.

Proof. $\infty L$ shall denote the representation of $W$ defined as follows. The representation space, $\mathfrak{F}(\infty L)$, will be the collection of all sequences $\left\{\psi_{i}\right\}$ of elements of $\mathfrak{H C}(L)$ for which $\sum_{i}\left\|\psi_{i}\right\|^{2}<\infty$ and we define $(\infty L)_{x}\left\{\psi_{i}\right\}=\left\{L_{x} \psi_{i}\right\}$ for all $x \in W$.

Suppose first that $L \sim M$. There exist algebraic isomorphisms, $\varphi_{1}$ and $\varphi_{2}$ respectively, of $a(L)$ onto $a(\infty L)$ and $a(\infty M)$ onto $a(M)$ such that $\varphi_{1}\left(L_{x}\right)=(\infty L)_{x}$ and $\varphi_{2}\left((\infty M)_{x}\right)=M_{x}$ for all $x \in w$. The easy verification that $\varphi_{1}$ and $\varphi_{2}$ defined in this way may be extended to algebraic isomorphisms of $a(L)$ onto $Q(\infty L)$ and $a(\infty M)$ onto $Q(M)$ respectively, is left to the reader.

Since $L \sim M,[14$, Lemma 1.5$]$ implies that $\infty L \simeq \infty M$. Thus, by definition, there exists a unitary transformation $U$ of $\mathcal{H C}(\infty L)$ onto $\mathfrak{H C}(\infty M)$ such that $U(\infty L)_{x} U^{-1}=(\infty M)_{x}$ for all $x \in w$. Thus we have a spatial isomorphism $\varphi_{3}$ of $Q(\infty L)$ onto $Q(\infty M)$ such that $\varphi_{3}\left((\infty L)_{x}\right)=(\infty M)_{x}$ for all $x \in W$. Hence $\varphi=\varphi_{2} \circ \varphi_{3} \circ \varphi_{1}$ is an algebraic isomorphism of $Q(L)$ onto $Q(M)$ such that $\varphi\left(L_{x}\right)=M_{x}$ for all $x \in W$.

Conversely, suppose such an isomorphism $\varphi$ exists. This then induces an isomorphism $\tilde{\varphi}$ of $a(\infty L)$ onto $a(\infty M)$ such that $\tilde{\varphi}\left((\infty L)_{x}\right)=(\infty M)_{x}$ for all $x \in W$. Consider the collection of projections $\left\{E_{i}\right\} \subset R(\infty L, \infty L)$ defined by $E_{i}\left(\psi_{1}, \psi_{2}, \cdots\right)=\left(0,0, \cdots, 0, \psi_{i}, 0, \cdots\right)$ where $\left(\psi_{1}, \psi_{2}, \cdots\right) \in \mathcal{H C}(\infty L)$ and $\psi_{j} \in \mathcal{H}(L), i, j,=1,2, \cdots$, and the collection of projections $\left\{F_{i}\right\}$ $\subset \Re(\infty M, \infty M)$ defined similarly, i.e., $F_{i}$ is the projection on $\mathfrak{H C}(\infty M)$ which maps a vector into its $i$ th coordinate. Then the collection $\left\{E_{i}\right\}$ (respectively $\left.\left\{F_{i}\right\}\right)$ is an infinite collection of mutually equivalent, two-by-two orthogonal projections whose sum is the identity on $\mathfrak{H}(\infty L)$ (respectively $\mathfrak{H}(\infty M)$ ). Hence, by $[1$, Corollary 7, p. 321], the isomorphism $\tilde{\varphi}$ of $\mathscr{Q}(\infty L)$ onto $a(\infty M)$ is spatial. Thus $\infty L \simeq \infty M$. Hence $M\} \infty M \simeq \infty L \geqq L$ and thus $M\} L$. Similarly $L\} M$. Hence $L \sim M$.

LEMMA 5. Let $L$ and $M$ denote unitary representations of a separable locally compact group $G$ and let $L^{\prime}$ and $M^{\prime}$ denote the corresponding nowhere trivial 
*-representations of the group algebra $a_{G}$. Then $L \sim M$ if and only if $L^{\prime} \sim M^{\prime}$. Indeed let $\varphi$ denote an isomorphism of $a(L)$ onto $Q(M)$. Then $\varphi\left(L_{x}\right)=M_{x}$ for all $x$ in $G$ if and only if $\varphi\left(L_{f}^{\prime}\right)=M_{f}^{\prime}$ for all $f$ in $Q_{G}$.

Proof. We first remark that the above statement is well-known in the case where $\varphi$ is a spatial isomorphism. Lemma 5 is then just the known fact that $L \simeq M$ if and only if $L^{\prime} \simeq M^{\prime}$.

Let $\varphi_{1}$ denote the obvious algebraic isomorphism of $a(L)=a\left(L^{\prime}\right)$ onto $a(\infty L)=a\left(\infty L^{\prime}\right)$. (Note that $(\infty L)^{\prime}=\infty L^{\prime}$.) Similarly let $\varphi_{2}$ denote the obvious algebraic isomorphism of $a(M)=a\left(M^{\prime}\right)$ onto $a(\infty M)=a\left(\infty M^{\prime}\right)$. Then, as we remarked in the proof of Lemma $4, \varphi^{\prime}=\varphi_{2} \circ \varphi \circ \varphi_{1}^{-1}$ is a spatial isomorphism of $a(\infty L)$ onto $a(\infty M)$. Since the statement is true for spatial isomorphisms, we have that $\varphi^{\prime}\left(\infty L_{x}\right)=\infty M_{x}$ for all $x$ in $G$ if and only if $\varphi^{\prime}\left(\infty L_{f}^{\prime}\right)=\infty M_{f}^{\prime}$ for all $f$ in $Q_{G}$. It thus follows that $\varphi=\varphi_{2}^{-1} \circ \varphi^{\prime} \circ \varphi_{1}$ has the property that $\varphi\left(L_{x}\right)=M_{x}$ for all $x$ in $G$ if and only if $\varphi\left(L_{f}^{\prime}\right)=M_{f}^{\prime}$ for all $f$ in $a_{G}$.

We continue to prove a couple of additional lemmas. The terminology of "measurable fields of vectors" (or "of operators," or "of von Neumann algebras") is that of Dixmier and the reader is referred to [1] for the definitions of these and other terms to be used in the following paragraphs.

Lemma 6. Let $(S, \mu)$ be a measure space and, for each $n$, let $y \rightarrow T_{n}^{\nu}$ be a measurable field of operators on $S$. Further suppose that, for all $n, T_{n}^{v}$ acts on the same Hilbert space $\mathcal{H}(y)$ and that $T_{n}^{y}$ converges to $T^{y}$ in the strong operator topology, for $\mu$-almost all $y$. Then $y \rightarrow T^{y}$ is a measurable field of operators.

Proof. Let $\left\{\psi_{1}, \psi_{2}, \cdots\right\}$ denote a fundamental sequence of measurable fields of vectors having values in $\mathfrak{H C}(y)$. Then by $[1$, Proposition $1, \mathrm{p} .157]$ we have that, for each $n$, the functions $y \rightarrow\left(T_{n}^{\nu} \psi_{i}(y), \psi_{j}(y)\right)$ are measurable. Since $T_{n}^{y} \rightarrow T^{y}$ in the strong and thus in the weak topology for $\mu$-almost all $y$, we have that the functions $\left(T_{n}^{y} \psi_{i}(y), \psi_{j}(y)\right)$ converge to $\left(T^{y} \psi_{i}(y), \psi_{j}(y)\right)$ pointwise $\mu$-almost everywhere. Hence the functions $y \rightarrow\left(T \psi_{i}(y), \psi_{j}(y)\right)$ are measurable and the lemma is proved.

Lemma 7. Let $L$ denote a nowhere trivial *-representation of a separable Banach *-algebra $\mathfrak{A}$. If $T \in Q(L)$, then there exists a sequence of operators $\left\{T_{i}\right\}$ contained in the range of $L$ such that $\left\|T_{i}\right\| \leqq\|T\|$ for all $i$ and the sequence $\left\{T_{i}\right\}$ converges to $T$ in the strong operator topology.

Proof. The range $R$ of $L$ is a *-algebra of operators which, by [1, Corollary 1 to Theorem 2, p. 44], is everywhere dense in $a(L)$, in the strong topology. Let $m=\|T\|$. Then by Kaplansky's density theorem [1, Theorem 3, p. 46] the ball $R_{m}$ of $R$ of radius $m$, is everywhere dense, in the strong topology, in $a_{m}$, the ball of $a(L)$ of radius $m$. Thus $T$ is contained in the strong closure of $R_{m}$. By [1, corollary to Proposition 1, p. 33], there exists a sequence of elements in $R_{m}$ which converges strongly to $T$. 
Proposition 5. Suppose $L$ and $M$ are representations of w with central decompositions over the same measure space, say $L=\int_{S} L^{\nu} d \mu(y)$ and $M=\int_{S} M^{y} d \mu(y)$. If $L^{\nu} \sim M^{y}$ for $\mu$-almost all $y$, then $L \sim M$.

Proof. We first restrict ourselves to the special case where $W$ is a Banach *-algebra and $L$ and $M$ are nowhere trivial *-representations of $W$. By Lemma 4 we have, for $\mu$-almost all $y$ in $S$, that there exists an algebraic isomorphism $\varphi_{y}$ of $a\left(L^{y}\right)$ onto $Q\left(M^{y}\right)$ such that $\varphi_{y}\left(L_{x}^{\nu}\right)=M_{x}^{y}$ for all $x$ in $w$. We next show that $y \rightarrow \varphi_{y}$ is a measurable field of homomorphisms. This means that if $y \rightarrow T^{v} \in Q\left(L^{y}\right)$ is a measurable field of operators, then $y \rightarrow \varphi_{y}\left(T^{v}\right)$ $\in Q\left(M^{y}\right)$ is a measurable field of operators. If $y \rightarrow T^{v} \in Q\left(L^{v}\right)$ is a measurable field of operators, we may form $T=\int_{S} T^{\nu} d \mu(y)$ and by Proposition 4, $T \in Q(L)$. By Lemma 7 there exists a sequence of elements, say $L_{x_{i}}$ in the range of $L$ such that $L_{x_{i}}$ converges to $T$ is the strong topology and furthermore $\left\|L_{x_{i}}\right\|$ $\leqq\|T\|$ for all $i$. Further each $L_{x_{i}}$ is decomposable and therefore we may write $L_{x_{i}}=\int_{S} L_{x_{i}}^{y} d \mu(y)$, where, for each $i, y \rightarrow L_{x_{i}}^{y}$ is a $\mu$-measurable field of operators. Further, by $\left[1\right.$, Proposition 4(i), p. 162], there exists a subsequence $L_{\boldsymbol{x}_{\boldsymbol{i}_{k}}}$ of $L_{x_{i}}$ such that $L_{x_{i_{k}}}$ converges to $T$ in the strong topology and further, for $\mu$ almost all $y, L_{x_{i k}}^{\nu}$ converges to $T^{v}$ in the strong topology. Thus we may assume, without loss of generality, that we have a sequence $L_{x_{i}}$ such that $L_{x_{i}}$ converges to $T$ and $L_{x_{i}}^{\nu}$ converges to $T^{\nu}$ for $\mu$-almost all $y$, in the strong topology. Now for each $i, \varphi_{y}\left(L_{x_{i}}^{v}\right)=M_{x_{i}}^{v}$ and thus, for each $i, y \rightarrow M_{x_{i}}^{v}$ is a measurable field of operators. By [1, Corollary 1, p. 57], $\varphi_{y}$ is strongly continuous on bounded sets. Since the sequence $L_{x_{i}}^{v}$ is a bounded sequence we have that $\varphi_{\nu}\left(L_{x_{i}}^{\nu}\right)$ converges strongly to $\varphi_{y}\left(T^{\nu}\right)$ for $\mu$-almost all $y$. Hence, by Lemma 6 , $y \rightarrow \varphi_{y}\left(T^{y}\right)$ is a measurable field of operators.

We are now in a position to define the map $\varphi$ of $a(L)$ onto $a(M)$. If $T=\int_{S} T^{v} d \mu(y) \in Q(L)$, let $\varphi(T)=\int_{S} \varphi_{y}\left(T^{\nu}\right) d \mu(y) \in Q(M)$. Then, by [1, Proposition 10, p. 193], $\varphi$ is an algebraic isomorphism of $Q(L)$ onto $Q(M)$. Further $\varphi\left(L_{x}\right)=\int_{S} \varphi_{y}\left(L_{x}^{v}\right) d \mu(y)=\int_{S} M_{x}^{v} d \mu(y)=M_{x}$ for all $x$ in $W$. By Lemma 4 we have $L \sim M$.

Since every ${ }^{*}$-representation of $W$ is unitarily equivalent to a nowhere trivial *-representation of $W$, it is clear that this result holds whenever $L$ and $M$ are *-representations of $w$.

Suppose now that $W=G$ is a separable locally compact group and $L$ and $M$ are unitary representations of $G$. Since $L^{y} \sim M^{y}$ for $\mu$-almost all $y$, we have, by Lemma 4 , that there exists isomorphisms $\varphi_{y}$ of $Q\left(L^{y}\right)$ onto $Q\left(M^{y}\right)$ such that $\varphi_{y}\left(L_{x}^{\nu}\right)=M_{x}^{\nu}$ for all $x$ in $G$. Let $\left(L^{y}\right)^{\prime}$ and $\left(M^{y}\right)^{\prime}$ denote the ${ }^{*}$-representations of the group algebra $a_{G}$ corresponding to $L^{y}$ and $M^{y}$ respectively. By Lemma 5 we have $\varphi_{y}\left(\left(L^{y}\right)_{f}^{\prime}\right)=\left(M^{y}\right)_{f}^{\prime}$ for all $f$ in $a_{G}$ and for $\mu$-almost all $y$. We may now apply the procedure used above to verify that $y \rightarrow \varphi_{y}$ is a measurable field of isomorphisms. Thus $\varphi=\int_{S} \varphi_{\nu} d \mu(y)$ is an isomorphism of $a(L)$ onto $a(M)$ such that $\varphi\left(L_{x}\right)=M_{x}$ for all $x$ in $G$. Hence, by Lemma $4, L \sim M$.

Proposition 6. Suppose that $L$ and $M$ are quasi-equivalent representations 
of w. Then $L$ and $M$ have central decompositions over the same measure space, $L=\int_{S} L^{y} d \mu(y)$ and $M=\int_{S} M^{y} d \mu(y)$ such that $L^{y} \sim M^{y}$ for $\mu$-almost all $y$.

Proof. Let $L=\int_{S} L^{y} d \mu(y)$ denote the central decomposition of $L$. Let $\varphi$ denote the isomorphism of $\mathfrak{Q}(L)$ onto $Q(M)$ given by Lemma 4. By $[1$, Proposition 11, p. 193], we may express $Q(M)$ in the form $Q(M)=\int_{S} \beta(y) d \mu(y)$, where $y \rightarrow B(y)$ is a $\mu$-measurable field of von Neumann algebras. Further there exists a $\mu$-measurable field of isomorphisms $y \rightarrow \varphi_{\nu}$ such that $\varphi_{\nu}$ maps $a\left(L^{y}\right)$ onto $\beta(y)$. Finally we have $\varphi=\int_{S} \varphi_{y} d \mu(y)$. For $\mu$-almost all $y$, we may define the representation $x \rightarrow M_{x}^{\nu}$ by $M_{x}^{\nu}=\varphi_{y}\left(L_{x}^{\nu}\right)$. Since $y \rightarrow \varphi_{y}$ is $\mu$-measurable, it is easy to verify that $M=\int_{S} M^{y} d \mu(y)$. By Lemma 4 we have $L^{y} \sim M^{y}$ for $\mu$-almost all $y$. It remains to verify that the decomposition, $M=\int_{S} M^{v} d \mu(y)$ constructed in this way, is indeed the central decomposition. To do this we use a criterion given in [14, Lemma 1.1]. The decomposition $M=\int_{S} M^{y} d \mu(y)$ of $M$ into primary representations is the central decomposition if and only if, for every Borel subset $B$ of $S$, we have $\int_{B} M^{y} d \mu(y) \succ \int_{S-B} M^{y} d \mu(y)$. We proceed to prove that this condition is satisfied. Almost every $M^{y}$ is primary since each $L^{y}$ is primary and $L^{y} \sim M^{y}$ for $\mu$-almost all $y$. Let $B$ denote a Borel subset of $S$ and let $L^{1}=\int_{B} L^{y} d \mu(y), L^{2}=\int_{S-B} L^{y} d \mu(y), M^{1}=\int_{B} M^{y} d \mu(y)$ and $M^{2}=\int_{S-B} M^{y} d \mu(y)$. By the criterion stated above we have $L^{1} \delta L^{2}$. Further $\varphi_{1}=\int_{B} \varphi_{\nu} d \mu(y)$ is an isomorphism of $Q\left(L^{1}\right)$ onto $Q\left(M^{1}\right)$ such that $\varphi_{1}\left(L_{x}^{1}\right)=M_{x}^{1}$ for all $x$ in $W$ and thus $L^{1} \sim M^{1}$. Similarly $L^{2} \sim M^{2}$. Hence $M^{1} \circ M^{2}$ and, by the criterion stated above, $M=\int_{S} M^{y} d \mu(y)$ is the central decomposition of $M$.

The last two propositions may be combined to give us our third theorem.

THEOREM 3. Two representations $L$ and $M$ of W are quasi-equivalent if and only if, when their central decompositions are taken over the quasi-dual $\tilde{\mathbb{W}}$ according to Theorem 2, they determine the same standard measure class $\mu$ on $\tilde{\mathfrak{W}}$.

5. The canonical lattice ideal of measure classes on $\tilde{\mathcal{W}}$. Theorem 3 establishes a one-to-one correspondence between $Q$ and a certain collection of measure classes on $\tilde{\mathscr{W}}$. We shall call this collection the canonical lattice ideal of measure classes on $\tilde{\mathscr{W}}$, and denote it $\operatorname{CrT}(\tilde{\mathbb{W}})$. The elements of $\operatorname{erT}(\tilde{\mathbb{W}})$ will be called canonical measure classes. To justify the terminology we must, of course, show that $\operatorname{err}(\tilde{\mathscr{W}})$ is a lattice ideal of the lattice $\operatorname{Tr}(\tilde{\mathscr{W}})$ of all standard measure classes on $\tilde{\mathbb{W}}$. For convenience, we shall adopt the following convention. If a Greek letter, say $\mu$, denotes a measure class, then that letter with a bar, say $\bar{\mu}$, shall always mean some measure contained in the given measure class $\mu$. The partial ordering in $\mathfrak{T}(\mathscr{W})$ is given by absolute continuity. Thus for $\mu, \nu \in \mathscr{T}(\tilde{\mathbb{W}})$, we write $\mu \leqq \nu$ if, whenever $B$ is a Borel subset of $\tilde{\mathbb{W}}$ such that $\bar{\nu}(B)=0$, then $\bar{\mu}(B)=0$. We say two elements $\mu$ and $\nu$ of $\mathfrak{T}(\tilde{\mathbb{W}})$ are disjoint, denoted $\mu \perp \nu$, if there exists a Borel subset $B$ of $\tilde{\mathcal{W}}$ such that $\bar{\mu}(B)=0$ and $\bar{\nu}(\tilde{\mathbb{W}}-B)=0$. Sometimes the term "orthogonal" is used in place of the term "disjoint." The join, $\mu \bigvee \nu$, of two elements of $\mathfrak{T}(\tilde{\mathbb{W}})$ is defined in the usual manner. The fact that $\operatorname{erT}(\tilde{\mathscr{W}})$ is indeed a lattice ideal of $\mathfrak{T}(\tilde{\mathscr{W}})$ will follow from the following theorem. 
THEOREM 4. The one-to-one correspondence between the elements of $Q$ and elements of $\mathfrak{M}(\tilde{\mathbb{W}})$ given by Theorems 2 and 3 is order preserving. That is to say, if $L, M \in Q$, then $L\} M$ if and only if $\mathfrak{e}(L) \geqq \mathfrak{C}(M)$, where $\mathfrak{C}(L)$ and $\mathfrak{C}(M)$ denote the measure classes on $\tilde{\mathcal{W}}$ corresponding to $L$ and $M$ respectively.

Proof. Suppose first that $L$ covers $M$. Then it is well known (see Proposition 1 and its proof) that $L$ may be written $L \simeq L^{1}+L^{2}$ where $L^{1} \sim M$ and $L^{2} \delta M$ and $L^{1} \delta L^{2}$. Suppose $L \simeq \int \tilde{\widetilde{W}} L^{y} d \bar{\mu}(y)$, where $\mu=\mathfrak{C}(L)$. Then since $L^{1} \circ L^{2}$, there exists a projection $E$ in $\mathcal{C} R(L)$ such that $L^{1}=L E$ and $L^{2}$ $=L(I-E)$. Since the above decomposition is central, $E$ is in the range of the corresponding projection valued measure. Thus there exists a Borel subset $B \subset \tilde{\mathscr{W}}$ such that $L^{1} \simeq \int_{B} L^{y} d \bar{\mu}(y)$ and $L^{2} \simeq \int(\tilde{\mathbb{W}}-B) L^{y} d \bar{\mu}(y)$. Define the measure $\pi$ on $\tilde{W}$ by $\pi(C)=\bar{\mu}(C \cap B)$ for all Borel sets $C$ in $\tilde{\mathcal{W}}$. Then $L^{1}$ has central decomposition $L^{1} \simeq \int \tilde{\mathscr{W}} L^{y} d \pi(y)$. Clearly $\bar{\mu} \geqq \pi$. However, since $L^{1} \sim M$ we have, by Theorem 3 , that $\pi \simeq \bar{\nu}$ where $\nu=\mathfrak{C}(M)$. Hence $\mu \geqq \nu$.

Conversely, suppose $\mu \geqq \nu$, where $\mu=\mathcal{C}(L)$ and $\nu=\mathfrak{C}(M)$. Then we may write $\mu=\mu_{1} \bigvee \mu_{2}$ where $\mu_{1} \simeq \bar{\nu}, \mu_{2} \perp \nu$ and $\mu_{1} \perp \mu_{2}$. Indeed, there exists a Borel set $B \subset \tilde{\mathbb{W}}$ such that $\bar{\mu}_{1}(C)=\bar{\mu}(C \cap B)$ and $\bar{\mu}_{2}(C)=\bar{\mu}(C \cap(\tilde{\mathbb{W}}-B))$ for all Borel sets $C \subset \tilde{\mathscr{W}}$. Cf. [9, Theorem 2, p. 78]. Then $L \simeq \int \tilde{W}_{\mathscr{W}} L y d \bar{\mu}(y)=\int \tilde{\mathscr{W}}^{y} L^{y} d \bar{\mu}_{1}(y)$ $+\int \tilde{\mathscr{W}}^{v} L^{v} \bar{\mu}_{2}(y)$. According to Theorem $3, \bar{\mu}_{1} \sim \bar{\nu}$ implies that $\int \tilde{\mathscr{W}}^{v} L^{v} \bar{\mu}_{1}(y)$ $\sim \int \tilde{\mathbb{W}^{\nu}} M^{v} d \bar{\nu}(y) \simeq M$. Hence $\left.L\right\} M$.

COROLlaRy 1. $\operatorname{erT}(\tilde{\mathbb{W}})$ is a $\sigma$-complete lattice ideal.

Proof. Clearly if $\mu$ and $\nu$ are in $\operatorname{eNT}(\tilde{\mathscr{W}})$, then $\mu \vee \nu \in \mathcal{C N}(\tilde{\mathscr{W}})$. Suppose that $\mu \in \operatorname{CIN}(\tilde{\mathbb{W}})$ and that $\nu \leqq \mu$. Then there exists a Borel subset $B \subset \tilde{\mathbb{W}}$ such that $\bar{\mu}_{B} \simeq \bar{\nu}$ where $\bar{\mu}_{B}$ is defined on $\tilde{Q}$ by $\bar{\mu}_{B}(C)=\bar{\mu}(B \cap C)$ for all Borel subsets $C$ of $\tilde{\mathcal{W}}$. Further there exists a representation $L$ of $\tilde{\mathbb{W}}$ such that $L \simeq \int \tilde{\mathscr{W}} L^{y} d \bar{\mu}(y)$ and $L^{1}=\int_{B} L^{y} d \bar{\mu}(y)=\int \tilde{\mathscr{W}} L^{y} d \bar{\mu}_{B}(y)$ is a subrepresentation of $L$. Hence $\mu_{B}$ $\in \operatorname{err}(\tilde{\mathscr{W}})$ and, since $\bar{\mu}_{B} \simeq \bar{\nu}, \nu \in \operatorname{CrT}(\tilde{\mathscr{W}})$. Thus $\operatorname{err}(\tilde{\mathscr{W}})$ is a lattice ideal. The correspondence of Theorem 4 is then a lattice isomorphism between $Q$ and $\operatorname{err}(\tilde{\mathscr{W}})$. By Proposition 1, $Q$, and thus $\operatorname{erl}(\tilde{\mathbb{W}})$, is $\sigma$-complete.

CoROllary 2. The lattice $Q$ of all quasi-equivalence classes of representations of $w$ is boundedly complete. More explicitly, every bounded collection $\left\{L^{\alpha}\right\}$ of elements of $Q$ has a supremum, and, in fact $\left\{L^{\alpha}\right\}$ has a countable subfamily $\left\{L^{\alpha_{i}}\right\}$ such that $\mathrm{V}_{\alpha} L^{\alpha}=\mathrm{V}_{i} L^{\alpha_{i}}$.

Proof. [9, Theorem 1, p. 79] may be used to show that $\mathcal{C}$ Tr( $(\tilde{W})$ has this property. Corollary 2 then follows since $Q$ and $\operatorname{erT}(\tilde{\mathscr{W}})$ are lattice isomorphic.

Corollary 3. Let $L$ and $M$ denote elements of $Q$, and $\mathfrak{e}(L)$ and $\mathfrak{e}(M)$ denote the corresponding measure classes on $\tilde{\mathcal{W}}$. Then $L \mathrm{\circ} M$ if and only if $\mathfrak{e}(L) \perp \mathfrak{e}(M)$.

We next point out that the canonical lattice ideal is, in general, a proper ideal of the lattice of all standard measures on $\tilde{\mathcal{W}}$. Indeed, consider the example given in [13, pp. 590-591] of a group $W$ which is the semi-direct product 
of the additive group of rational numbers $G_{1}$ with the multiplicative group of nonzero rational numbers $G_{2}$. Then the right regular representation $R$ of $w$, which is a type II primary representation, can be expressed as a direct integral of irreducible representations, $R \simeq \int_{\hat{G}_{2}} L^{y} d \mu(y)$ where $\hat{G}_{2}$ is the character group of $G_{2}$ and $\mu$ is Haar measure on $\hat{G}_{2}$. By [15, corollary to Theorem 8.7], $\hat{G}_{2}$ is smooth and hence by [7, Theorem 2], $\hat{G}_{2}$ is a standard Borel space. Further the components in this decomposition are two-by-two disjoint. Thus we may proceed, by the method used in the proof of Theorem 2, to obtain a standard $\sigma$-finite Borel measure $\tilde{\mu}$ on $\tilde{\mathbb{W}}$ such that $R \simeq \int \tilde{\mathbb{W}} L^{y} d \mu(y)$. This is clearly not the central decomposition, since the central decomposition of a primary representation is the trivial decomposition. Thus $\tilde{\mu}$ is not contained in $\operatorname{CIT}(\tilde{\mathscr{W}})$. However if $\tilde{\mathscr{W}}$ is type $\mathrm{I}$, then $\operatorname{CMT}(\tilde{\mathscr{W}})$ is identical with the lattice of all standard measure classes on $\tilde{\mathscr{W}}$.

In the nontype I case a new aspect of the representation problem, which has no analogue in the classical theory, is forced upon us. If we are to classify all representations of a nontype I group or *-algebra we must not only determine the dual object $\tilde{\mathcal{W}}$, but we must also characterize the canonical lattice ideal of measure classes on $\tilde{W}$. The natural question as to the difficulties involved in this new aspect of the representation problem cannot be properly answered until more extensive research is done in the still embryonic field of representations of nontype I groups. We are able, however, to give a superficial, but perhaps useful, characterization of the canonical lattice ideal $\operatorname{err}(\mathscr{W})$, which we now proceed to describe.

We define a $Q$-valued measure $\mu$ on $\tilde{\mathbb{W}}$ to be a function on the Borel sets of $\tilde{\mathbb{W}}$ which assigns to each Borel set $B$ of $\widetilde{\mathscr{W}}$, a quasi-equivalence class $\mu(B)$, of representations of $W$. Recalling that $Q$ is a $\sigma$-complete lattice (Proposition 1) we also require $\mu\left(\mathrm{U}_{n} B_{n}\right)=\mathrm{V}_{n} \mu\left(B_{n}\right)$ whenever $\left\{B_{n}\right\}$ is a countable collection of Borel subsets of $\tilde{\boldsymbol{W}}$.

Let $\mu$ denote a standard measure class on $\tilde{\mathscr{W}}$ and suppose that there exists a $\mu$-measurable map $y \rightarrow L^{y}$ of $\tilde{\mathcal{W}}$ into $W^{c}$ such that $L^{y} \in y$ for $\mu$-almost all $y$. From this we may construct a $Q$-valued measure $\mu^{\prime}$ on $\tilde{\mathbb{W}}$ as follows. For each Borel set $B \subset \tilde{W}$, let $\mu^{\prime}(B)$ denote the quasi-equivalence class containing $\int_{B} L^{y} d \mu(y)$. The reader may verify that $\mu^{\prime}$ is then a $Q$-valued measure. By Proposition 5 , if $\mu$ is a canonical measure class, then the corresponding $Q$ valued measure $\mu^{\prime}$ does not depend on the mapping $y \rightarrow L^{y}$ of $\tilde{W}$ into $W^{c}$. Recall that one may take relative complements in $Q$ (see Proposition 1). Thus we may define a $Q$-valued measure to be subtractive if, whenever $B_{1}$ and $B_{2}$ are Borel subsets of $\tilde{\mathcal{W}}$ such that $B_{1} \subset B_{2}$, then $\mu\left(B_{2}-B_{1}\right)=\mu\left(B_{2}\right)-\mu\left(B_{1}\right)$. We are now in a position to state our characterizations of $\operatorname{err}(\tilde{\mathbb{W}})$.

Proposition 7. A standard measure class $\mu$ on $\tilde{\mathbb{W}}$ is a member of $\operatorname{CTr}(\tilde{\mathbb{W}})$ if and only if (a) there exists a $\mu$-measurable map $y \rightarrow L^{y}$ of $\tilde{W}$ into $W^{c}$ such that $L^{y} \in y$ for $\mu$-almost all $y$ and $(\mathrm{b})$ the corresponding $Q$-valued measure $\mu^{\prime}$ on $\tilde{W}$ is subtractive. 
Proof. Suppose that $\mu$ is contained in $\operatorname{CrT}(W)$. Then there exists a $\mu$ measurable map $y \rightarrow L^{y}$ of $\tilde{W}$ into $\mathscr{W}^{c}$ such that $L^{y} \in y$ and let $\mu^{\prime}$ be the corresponding $Q$-valued measure on $\tilde{\mathcal{W}}$. Let $B_{1}, B_{2}$ denote Borel subsets of $\tilde{\mathscr{W}}$ such that $B_{1} \subset B_{2}$. Then $B_{1} \cap\left(B_{2}-B_{1}\right)$ is empty and thus $\int_{B_{1}} L^{y} d \mu(y)$ of $\int_{\left(B_{2}-B_{1}\right)} L^{y} d \mu(y)$ which implies that $\mu^{\prime}\left(B_{1}\right)$ o $\mu^{\prime}\left(B_{2}-B_{1}\right)$. Since $\mu^{\prime}\left(B_{2}\right)$ $=\mu^{\prime}\left(B_{1} \cup\left(B_{2}-B_{1}\right)\right)=\mu^{\prime}\left(B_{1}\right) \bigvee \mu^{\prime}\left(B_{2}-B_{1}\right)$, we have, by the definition of "relative complement," that $\mu^{\prime}\left(B_{2}-B_{1}\right)=\mu^{\prime}\left(B_{2}\right)-\mu^{\prime}\left(B_{1}\right)$.

Conversely, suppose $\mu$ is a standard measure class on $\tilde{\mathbb{W}}$, that $y \rightarrow L^{y}$ is a $\mu$-measurable map of $\tilde{W}$ into $W^{c}$ such that $L^{y} \in y$ and that the corresponding $Q$-valued measure $\mu^{\prime}$ is subtractive. Let $B$ denote a Borel subset of $\tilde{\mathcal{W}}$. Then $\mu^{\prime}(\tilde{\mathscr{W}}-B)=\mu^{\prime}(\tilde{\mathbb{W}})-\mu^{\prime}(B)$ and thus $\mu^{\prime}(\tilde{\mathscr{W}}-B) \delta \mu^{\prime}(B)$. Hence $\int(\tilde{\mathscr{W}}-B) L^{y} d \mu(y)$ $\delta \int_{B} L^{y} d \mu(y)$ for every Borel set $B \subset \tilde{\mathscr{W}}$. Hence, by [14, Lemma 1.1], the range of the projection valued measure associated with the decomposition $\int \tilde{\widetilde{W}} L^{y} d \mu(y)$ is contained in the center $\mathcal{C} R(L)$ of the commuting algebra, $\Re(L, L)$, of $L$. However this is a decomposition in which all the components are primary representations, which is therefore a refinement of the central decomposition. Thus the range of the associated projection-valued measure contains, and thus is identical with, the set of all projections in $\mathcal{C} R(L)$. Hence the decomposition is central and $\mu \in \operatorname{CIr}(\tilde{\mathbb{W}})$.

6. Multiplicity theory. Up to this point we have been concerned with classifying representations up to quasi-equivalence, while traditionally the problem is to determine all representations up to unitary equivalence. It is therefore necessary to distinguish, up to unitary equivalence, the different representations contained in a given quasi-equivalence class. This is the purpose of multiplicity theory. Such a theory is already well known. For example $[14$, Theorem 1.5] gives a "relative" multiplicity theory for the type II case. Let $L$ denote a type II quasi-equivalence class and let $M$ denote any finite representation contained in this class. (A representation $T$ is called infinite if $T \simeq \infty T . T$ is called finite if no subrepresentation of $T$ is infinite.) Then $[14$, Theorem 1.5] states that to each $\bar{L} \in L$, there is associated a projection $E$ in $\mathfrak{C} \mathfrak{R}(M)$ and a nonsingular self-adjoint operator $H$ in $\mathcal{C} R\left(M_{E}\right)$, where $M_{E}$ denotes the restriction of the representation $M$ to the range of $E$. Two representations in $L$ are then unitarily equivalent if and only if they correspond to the same projection $E$ and operator $H$.

We now proceed to develop a formulation of multiplicity theory which will mesh easily with our characterization of the lattice of quasi-equivalence classes. Since we now have a lattice of measure classes before us, it seems natural to revert back to using old fashioned multiplicity functions.

Suppose $L \in Q$ and $\mu=\mathfrak{e}(L)$. Then we define a multiplicity function for $\mu$ to be any function $f$ defined on the lattice ideal of all $\sigma$-finite measure classes $\nu$ on $\tilde{W}$ such that $\nu \leqq \mu$, having non-negative real numbers and $\infty$ for values and satisfying the additional properties: (a) if $\nu=0$, then $f(\nu)=0$; (b) if $\nu_{1}$ and $\nu_{2}$ are $\sigma$-finite measure classes on $\tilde{\mathscr{W}}$ such that $0 \neq \nu_{1} \leqq \nu_{2} \leqq \mu$, then $f\left(\nu_{2}\right)$ 
$\leqq f\left(\nu_{1}\right)$; and (c) if a measure class $\nu$ is in the domain of $f$ and is the supremum of a countable family $\left\{\nu_{j}\right\}$ of two-by-two disjoint, nonzero measure classes on $\tilde{\mathcal{W}}$, then $f(\nu)=\inf \left\{f\left(\nu_{j}\right)\right\}$.

We next proceed to define a multiplicity function for $\mu$, corresponding to each representation $\bar{L} \in L$. [14, Theorem 1.2] states that $L$ may be expressed uniquely in the form $L=L_{\mathrm{I}} \bigvee L_{\mathrm{II}} \bigvee L_{\mathrm{III}}$ such that $L_{i} \delta L_{j}$ if $i \neq j$, and $L_{i}$ is either empty or $L_{i} \in Q$ and is of type $i$, for $i, j=\mathrm{I}$, II, III. Because the correspondence between $Q$ and $\operatorname{err}(\tilde{\mathscr{W}})$ is lattice preserving it follows that $\mu$ may be decomposed uniquely in the form $\mu=\mu_{\mathrm{I}} \bigvee \mu_{\mathrm{II}} \bigvee \mu_{\mathrm{III}}$ such that $\mu_{\boldsymbol{i}}=\mathfrak{e}\left(L_{\boldsymbol{i}}\right)$ and $\mu_{i} \perp \mu_{j}$ if $i \neq j, i, j=\mathrm{I}$, II, III. We call $\mu_{i}$ the type $i$ part of $\mu$, for $i=\mathrm{I}$, II, III.

Each $\bar{L} \in L$ then has a decomposition of the form $\bar{L} \simeq \int \tilde{w} L^{y} d \mu(y)$ $=\sum_{i=1}^{\text {III }} \int_{\tilde{W}_{\mathcal{W}}} L^{y} d \mu_{i}(y)$. Let $M$ denote any finite representation contained in $L_{\mathrm{II}}$, which then has a decomposition of the form $M \simeq \int \tilde{w} M^{y} d \mu_{\mathrm{II}}(y)$. By [14, Theorem 2.6], $L^{y}$ and $M^{y}$ are quasi-equivalent type II primary representations of $W$ of $\mu_{\mathrm{II}}$-almost all $y$ in $\tilde{W}$. Since $M$ is finite, each $M^{v}$ is finite for $\mu_{\mathrm{II}}$-almost all $y$ in $\tilde{\mathbb{W}}$. By the multiplicity theory for type II primary representations (cf. [14, p. 197]) $L^{y} \simeq m(y) M^{y}$ for $\mu_{\mathrm{II}}$-almost all $y$, where $m(y)$ is a positive real number or $\infty$. Then for $\nu \neq 0, \nu \leqq \mu_{\text {III }}$, we define

$$
f(\nu)=\nu \text {-ess. inf. }\{m(y)\}=\operatorname{Sup}\left\{m\left(y^{\prime}\right): y^{\prime} \in \tilde{W} \text { and } \nu\left\{y: m(y) \leqq m\left(y^{\prime}\right)\right\}=0\right\} \text {. }
$$

Similarly, by [14, Theorem 2.6], $L^{y}$ is a type I primary representation of W, for $\mu_{\mathrm{I}}$-almost all $y$ in $\tilde{W}$. Thus, by the multiplicity theory for type I primary representations, $L^{\boldsymbol{v}} \simeq n(y) N^{y}$, where $N^{y}$ is an irreducible representation, $N^{\nu} \in y$, and $n(y)$ is a positive integer or $\infty$, for $\mu_{\mathrm{I}}$-almost all $y$. If $\nu \neq 0$ and $\nu \leqq \mu_{\text {I }}$, we define $f(\nu)=\nu$-ess. inf. $\{n(y)\}$.

If $\nu \leqq \mu$, then $\nu=\nu_{\mathrm{I}} \bigvee \nu_{\mathrm{II}} \bigvee \nu_{\text {III }}$ where $\nu_{i} \leqq \mu_{i}$ for $i=\mathrm{I}$, II, III. We then define $f(\nu)=\inf \left\{f\left(\nu_{\mathrm{I}}\right), f\left(\nu_{\mathrm{II}}\right)\right\}$ if $\nu_{\mathrm{I}} \neq 0$ and $\nu_{\mathrm{II}} \neq 0, f(\nu)=0$ if $\nu=0, f(\nu)=f\left(\nu_{\mathrm{I}}\right)$ if $\nu_{\mathrm{I}} \neq 0$ and $\nu_{\mathrm{II}}=0, f(\nu)=f\left(\nu_{\mathrm{II}}\right)$ if $\nu_{\mathrm{I}}=0$ and $\nu_{\mathrm{II}} \neq 0$, and finally $f(\nu)=\infty$ if $\nu \neq 0, \nu_{\mathrm{I}}=0$ and $\nu_{\mathrm{II}}=0$.

Thus corresponding to each $\bar{L} \in L$ we associate a well-defined function on the lattice of measure classes absolutely continuous with respect to $\mu$ which, if the type II part $L_{\mathrm{II}}$ of $L$ is nonempty, depends on the choice of a finite representation $M$ in $L_{\text {II. }}$. For this reason we sometimes call this function a "relative multiplicity function." We next revise our notation in order to properly indicate the different objects upon which this function depends. $f_{\mu, M}(\bar{L}, \nu)$ will denote the function defined above, whose domain is the set $\{\nu: \nu \leqq \mu\}, \mu=\mathfrak{C}(L), \bar{L} \in L$, and $M$ is a finite representation in the type II part of $L$. The subscript $M$ may be suppressed in the case where $L_{\mathrm{II}}$ is empty. We leave to the reader the tedious task of verifying that, for fixed $\mu, M$ and $\bar{L}$, $f_{\mu, M}(\bar{L}, \cdot)$ is indeed a multiplicity function as defined above.

Our next task is to prove that this multiplicity function distinguishes representations, up to unitary equivalence, within a quasi-equivalence class. Before establishing this fact (Theorem 5) however, we must first obtain a couple of preliminary results. 
Proposition 8. Suppose $L \in Q, M \in Q, \bar{L} \in L, \bar{M} \in M, \mu=\mathfrak{e}(L)$, and $\nu=\mathfrak{C}(M)$. Let $\bar{L}=\int \tilde{\mathscr{W}} L^{y} d \mu(y)$ and $\bar{M}=\int \tilde{\mathscr{W}} M^{y} d \nu(y)$ represent the central decompositions of $\bar{L}$ and $\bar{M}$ respectively. Then $\bar{L} \leqq \bar{M}$ if and only if $\mu \leqq \nu$ and $L^{y} \leqq M^{y}$ for $\mu$-almost all $y$.

Proof. We remark first that in this proof and the material to follow, we adopt the usual practice of identifying representations which are unitarily equivalent, whenever it is convenient to do so.

Suppose first that $\bar{L} \leqq \bar{M}$. Then $L\left\{M\right.$ and $\nu=\mu \bigvee \mu^{\prime}$ where $\mu \perp \mu^{\prime}$. Let $M^{1}=\int \tilde{\mathscr{W}} M^{y} d \mu(y)$ and $M^{2}=\int \tilde{\mathscr{W}} M^{y} d \mu^{\prime}(y)$. Then $\bar{M}=M^{1}+M^{2}$ and $M^{2} \diamond \bar{L}$. Hence $\bar{L} \leqq M^{1}$. Then there exists a projection $E$ in $\mathcal{R}\left(M^{1}, M^{1}\right)$ such that $\bar{L}_{x}=E M_{x}^{1} E$ for all $x$ in $W$. By Corollary 2 to Proposition $4, E=\int \tilde{W} E^{y} d \mu(y)$, where $E^{y}$ is a projection in $R\left(M^{y}, M^{y}\right)$ for $\mu$-almost all $y$. By [1, Proposition 3, p. 161], $L_{x}^{y}=E^{y} M_{x}^{v} E^{y}$ for $\mu$-almost all $y$. Hence $L^{y} \leqq M^{y}$ for $\mu$-almost all $y$.

Conversely, suppose $\mu \leqq \nu$ and $L^{y} \leqq M^{y}$ for $\mu$-almost all $y$. Then we may write $\nu=\mu \bigvee \mu^{\prime}$ where $\mu \perp \mu^{\prime}$ and $\bar{M}=M^{1}+M^{2}$ as above. It is sufficient to prove $\bar{L} \leqq M^{1}$. Using the fact that $\mathfrak{H}\left(L^{y}\right)$ is an invariant subspace of $\mathscr{H}\left(M^{y}\right)$ for $\mu$ almost all $y$, the reader may verify directly from the definition of direct integral that $\mathcal{H}(\bar{L})$ is an invariant subspace of $\mathcal{H}\left(M^{1}\right)$ and that $\bar{L}$ is the restriction of $M^{1}$ to this subspace. Hence $\bar{L} \leqq \bar{M}$.

Let $L=\int \tilde{\mathcal{W}} L^{y} d \mu(y)$. Then if $L$ is type I, $L^{y}$ is a type I primary representation for $\mu$-almost all $y$. Thus if $N^{y}$ is an irreducible representation contained in the class $y$, we have $L^{y}=n(y) N^{y}$ where $n(y)$ is a positive integer or $\infty$, for $\mu$-almost all $y$. According to [14, Corollary 1 to Theorem 2.8], $y \rightarrow n(y)$ is a $\mu$-measurable function. We next prove the analogue of this result for the type II case.

Proposition 9. Let $L$ denote a type II representation with central decomposition $L=\int \tilde{\mathfrak{W}} L^{y} d \mu(y)$. Let $M$ denote a finite type II representation quasi-equivalent to $L$, with central decomposition $M=\int \tilde{\mathscr{W}} M^{y} d \mu(y)$. Then $L^{y}=m(y) M^{y}$ for $\mu$-almost all $y$, where, for each $y, m(y)$ is a positive real number or $\infty$. The function $y \rightarrow m(y)$ is $\mu$-measurable.

Proof. Given any $\lambda>0$, we may form the representation $M_{\lambda}=\int \tilde{\mathscr{W}} \lambda M^{y} d \mu(y)$. Further $L$ and $M_{\lambda}$ may each be decomposed into disjoint parts, say $L=L^{1}+L^{2}$ and $M_{\lambda}=M_{\lambda}^{1}+M_{\lambda}^{2}$, such that $L^{1} \leqq M_{\lambda}^{1}$ and $L^{2} \geqq M_{\lambda}^{2}$. (Cf. [14, p. 198] or [12, Theorem 1.13 , p. 24].) These decompositions are effected by a pair of projections $E$ and $F$ such that $E \in \mathbb{C} R(L)$ and $F \in \mathcal{C} R(M)$. Since $L \sim M_{\lambda}$ it follows that $L^{1} \sim M_{\lambda}^{1}$ and $L^{2} \sim M_{\lambda}^{2}$ and thus that $E$ and $F$ correspond to the same Borel set, say $B$ of $\tilde{\mathbb{W}}$. Hence by Proposition 8 we have $L^{y}=m(y) M^{y} \leqq \lambda M^{v}$ for $\mu$-almost all $y$ in $B$ and $L^{y}=m(y) M^{y} \geqq \lambda M^{y}$ for $\mu$-almost all $y$ in $(\tilde{W}-B)$.

Let $\left\{\lambda_{i}\right\}$ denote an increasing sequence of positive real numbers which approaches $\lambda$ from the left. Then for each $i$, there exists a measurable set $\mathfrak{N}_{i}$ such that $m(y) \leqq \lambda_{i}$ for all $y$ in $\mathfrak{N}_{i}$, and a measurable set $\mathfrak{N}_{i}^{\prime}$ such that

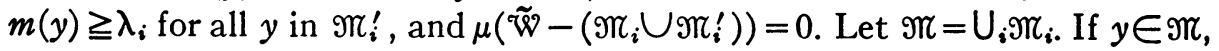


then $m(y) \leqq \lambda_{i}$ for some $i$ and thus $m(y)<\lambda$. Let $\mathscr{N}=\bigcup_{i}\left(\tilde{\mathscr{W}}-\left(\mathscr{T}_{i} \cup \mathscr{M}_{i}^{\prime}\right)\right)$. Then $\mu(\Re)=0$. Now suppose $m(y)<\lambda$ and $y \notin \Re$. If $y \in \Re_{i}^{\prime}$ for every $i$, then $\lambda_{i} \leqq m(y)$ for every $i$, which implies $m(y) \geqq \lambda$. Hence $y \notin \mathbb{N}_{i}^{\prime}$ for some $i$. Hence $y \in \Re$. Let $\Re_{1}$ denote the set of $y, y \in \tilde{W}$, for which $m(y)<\lambda$ and $y \notin \Re$. Then $\mathscr{N}_{1} \subset \mathscr{N}$ which implies $\mu\left(\mathscr{N}_{1}\right)=0$. Thus $\{y: m(y)<\lambda\}=\mathfrak{N}_{\mathcal{U}} \cup \mathscr{N}_{1}$ is a measurable set.

THEOREM 5. Let $\bar{L}_{1}$ and $\bar{L}_{2}$ denote two representations in the class $L$, where $L \in Q$. Let $\mu=\mathrm{e}(L)$ and let $M$ denote any finite representation contained in the type II part of $L$. Then $\bar{L}_{1} \leqq \bar{L}_{2}$ if and only if $f_{\mu, M}\left(\bar{L}_{1}, \nu\right) \leqq f_{\mu, M}\left(\bar{L}_{2}, \nu\right)$ for all $\nu \leqq \mu$.

Proof. Let $\bar{L}_{1}=\int \tilde{\mathscr{W}} L_{1}^{y} d \mu(y), \bar{L}_{2}=\int \tilde{\mathscr{W}} L_{2}^{y} d \mu(y)$, and $M=\int \tilde{\mathscr{W}} M^{y} d \mu_{\mathrm{II}}(y)$, where $\mu_{\mathrm{II}}$ is the type II part of $\mu$. If $L_{1}^{\nu}$ is type I, let $N^{y}$ denote an irreducible representation quasi-equivalent to $L_{1}^{\nu}$ and let $L_{1}^{y}=n_{1}(y) N^{y}$ and $L_{2}^{y}=n_{2}(y) N^{y}$. If $L_{1}^{\nu}$ is type II, let $L_{1}^{y}=m_{1}(y) M^{y}$ and $L_{2}^{y}=m_{2}(y) M^{y}$. This may be done for $\mu_{\mathrm{II}}{ }^{-}$ almost all $y$.

Suppose first that $\bar{L}_{1} \leqq \bar{L}_{2}$. By Proposition $8, L_{1}^{y} \leqq L_{2}^{y}$ for $\mu$-almost all $y$ and thus $n_{1}(y) \leqq n_{2}(y)$ for $\mu_{\mathrm{I}}$-almost all $y$, where $\mu_{\mathrm{I}}$ is the type I part of $\mu$. Suppose $\nu \leqq \mu$ and $\nu=\nu_{\mathrm{I}} \bigvee \nu_{\mathrm{II}} \bigvee \nu_{\mathrm{III}}$. Then

$$
f_{\mu, M}\left(\bar{L}_{1}, \nu_{\mathrm{I}}\right)=\nu_{\mathrm{I}} \text {-ess. inf. }\left\{n_{1}(y)\right\} \leqq \nu_{\mathrm{I}} \text {-ess. inf. }\left\{n_{2}(y)\right\}=f_{\mu, M}\left(\bar{L}_{2}, \nu_{\mathrm{I}}\right) .
$$

Similarly one may prove $f_{\mu, M}\left(\bar{L}_{1}, \nu_{\mathrm{II}}\right) \leqq f_{\mu, M}\left(\bar{L}_{2}, \nu_{\mathrm{II}}\right)$, which then gives $f_{\mu, M}\left(\bar{L}_{1}\right.$, $\nu) \leqq f_{\mu, M}\left(\bar{L}_{2}, \nu\right)$.

Conversely, suppose $f_{\mu, M}\left(\bar{L}_{1}, \nu\right) \leqq f_{\mu, M}\left(\bar{L}_{2}, \nu\right)$ for all $\nu \leqq \mu$. We shall prove $\bar{L}_{1} \leqq \bar{L}_{2}$ in two parts. We shall prove (a) that $n_{1}(y) \leqq n_{2}(y)$ for $\mu_{\mathrm{I}}$-almost all $y$, where $\mu_{\mathrm{I}}$ is the type I part of $\mu$ and (b )that $m_{1}(y) \leqq m_{2}(y)$ for $\mu_{\mathrm{II}}$-almost all $y$. (a) and (b) together then imply that $L_{1}^{y} \leqq L_{2}^{\nu}$ for $\mu$-almost all $y$, and thus, by Proposition 8 , that $\bar{L}_{1} \leqq \bar{L}_{2}$.

Part (a). Since $n_{1}(y)$ and $n_{2}(y)$ are measurable functions of $y$ (see [14, Corollary 1 to Theorem 2.8]) $B=\left\{y: n_{1}(y)>n_{2}(y)\right\}$ is a $\mu_{\mathrm{I}}$-measurable subset of $\tilde{\mathcal{W}}$. We assume that statement (a) above is false, i.e., we assume that $\mu_{\mathrm{I}}(B) \neq 0$. Let $\nu$ denote the restriction of $\mu_{\mathrm{I}}$ to $B$. Hence $f_{\mu, M}\left(\bar{L}_{1}, \nu\right)=\nu$-ess. inf. $\left\{n_{1}(y)\right\}>\nu$-ess. inf. $\left\{n_{2}(y)\right\}=f_{\mu, M}\left(\bar{L}_{2}, \nu\right)$, which is the desired contradiction. Thus $\nu_{\mathrm{I}}(B)=0$, and statement (a) above has been proved.

Part (b). Since $m_{1}(y)$ and $m_{2}(y)$ are $\mu_{\mathrm{II}}$-measurable functions of $y$ by Proposition 9, we have, for each positive integer $n$, that

$$
B_{n}=\left\{y: m_{2}(y)+1 / n<m_{1}(y)\right\}
$$

is a $\mu_{\mathrm{II}}$-measurable set. We proceed by way of contradiction and suppose that statement (b) is false, i.e., that the set $B=\left\{y: m_{2}(y)<m_{1}(y)\right\}$ is such that $\mu_{\mathrm{II}}(B) \neq 0$. Then for some positive integer, say $n=N, \mu_{\mathrm{II}}\left(B_{N}\right) \neq 0$. Let $\nu$ denote the restriction of $\mu_{\mathrm{II}}$ to the set $B_{N}$. Note that if $y \in B_{N}$, then $m_{2}(y) \neq \infty$. Then $\nu$-ess. inf. $\left\{m_{1}(y)\right\} \geqq \nu$-ess. inf. $\left\{m_{2}(y)+1 / N\right\}=\nu$-ess. inf. $\left\{m_{2}(y)\right\}+1 / N$ $>\nu$-ess. inf. $\left\{m_{2}(y)\right\}$. Hence $f_{\mu, M}\left(\bar{L}_{1}, \nu\right)>f_{\mu, M}\left(\bar{L}_{2}, \nu\right)$, which is the desired contradiction. Thus $\mu_{\mathrm{II}}(B)=0$ and statement (b) above has been proved. 
Corollary 1. Assume the hypothesis of Theorem 5. Then $\bar{L}_{1} \simeq \bar{L}_{2}$ if and only if $f_{\mu, M}\left(\bar{L}_{1}, \nu\right)=f_{\mu, M}\left(\bar{L}_{2}, \nu\right)$ for all $\nu \leqq \mu$.

Corollary 2. Let $\bar{L}_{1}$ and $\bar{L}_{2}$ denote any two representations of $W, L_{1}$ and $L_{2}$ the quasi-equivalence classes containing $\bar{L}_{1}$ and $\bar{L}_{2}$ respectively, and $\mu_{1}=\mathfrak{C}\left(L_{1}\right)$ and $\mu_{2}=\mathrm{e}\left(L_{2}\right)$. Let $M_{2}$ denote a finite representation contained in the type II part of $L_{2}$ and let $M_{1}$ denote the subrepresentation of $M_{2}$ having the property that $\left.\bar{L}_{1}\right\} M_{1}$ and $\left(\bar{L}_{2}-\bar{L}_{1}\right) \circ M_{1}$. Then $\bar{L}_{1} \leqq \bar{L}_{2}$ if and only if $\mu_{1} \leqq \mu_{2}$ and $f_{\mu_{1}, M_{1}}\left(\bar{L}_{1}, \nu\right)$ $\leqq f_{\mu_{2}, M_{2}}\left(\bar{L}_{2}, \nu\right)$ for all $\nu \leqq \mu_{1}$.

We conclude this section with a few remarks on the type I case. For a type I quasi-equivalence class $L$ the multiplicity function defined above is not "relative" and we may suppress the subscript $M$ in the notation $f_{\mu, M}(\bar{L}, \nu)$. Then we have that a representation $\bar{L}$ is multiplicity free if and only if $f_{\mu}(\bar{L}, \nu)=1$ for all $\nu \leqq \mu, \nu \neq 0$. A type I representation $\bar{L}$ is said to be uniformly of multiplicity $n$ if $\bar{L}=n M$, where $M$ is a multiplicity free representation. (Cf. $[12$, p. 41].) A measure $\mu$ is said to be uniformly of multiplicity $n$ relative to some multiplicity function $f$ which has $\mu$ in its domain if $f(\nu)=n$ for all $\nu \neq 0$ and $\nu \leqq \mu$. (Cf. $[9$, p. 81].) These two concepts are then related in the following manner.

Let $L$ denote a type I quasi-equivalence class, $\mu=\mathfrak{C}(L)$, and $\bar{L} \in L$. Then $\bar{L}$ is uniformly of multiplicity $n$ if and only if $\mu$ is uniformly of multiplicity $n$ relative to the multiplicity function $f_{\mu}(\bar{L}, \cdot)$.

We remark here that Theorem 1.4 of [14] may now be obtained as an easy consequence of well-known properties of multiplicity functions. Indeed, let $\bar{L}$ denote any type I representation. By $\left[9\right.$, Theorem 3, p. 82], $\mu=\mathrm{V}_{j} \mu_{j}$ where $\left\{\mu_{j}\right\}$ is a countable orthogonal family of measure classes such that each $\mu_{j}$ either has uniform multiplicity $j$, relative to the multiplicity function $f_{\mu}(\bar{L}, \cdot)$, or $\mu_{j}=0$, for $j=\infty, 1,2, \cdots$. Hence $\bar{L}$ may be decomposed $\bar{L}=\sum_{j} L_{j}$ where the components are two-by-two disjoint and $L_{j}$ is uniformly of multiplicity $j$. (Some terms may not appear.) But this is just [14, Theorem 1.4], except that the uniqueness of the decomposition has not been proven here.

7. Projective representations. We now restrict ourselves to the case where $W$ is a separable locally compact group. In certain branches of physics and in the study of induced representations it has been found necessary to enlarge the scope of representation theory to include a more general concept, that of "projective representation." A projective representation $L$ of a separable locally compact group $G$ is a mapping $x \rightarrow L_{x}$ of $G$ into the group of all unitary operators on a separable Hilbert space $\mathfrak{F}(L)$ such that (a) the image of the identity element $e$ of $G$ is the identity operator $I$, (b) for all $x$ and $y$ in $G, L_{x y}$ $=\sigma(x, y) L_{x} L_{y}$ where $\sigma(x, y)$ is a complex number depending on $x$ and $y$ and (c) the function $x \rightarrow\left(L_{x} \phi, \psi\right)$ is a Borel function on $G$ for each $\phi, \psi$ in $\operatorname{HC}(L)$. The function $(x, y) \rightarrow \sigma(x, y)$ is called the multiplier of $L$ and we refer to $L$ as a $\sigma$-representation. See $[16, \S 1]$, for the extensions of the usual concepts which are applicable to $\sigma$-representations. The importance of developing a 
decomposition theory which is not restricted to type I representations is greatly enhanced in this more general situation since there exist commutative groups which have primary $\sigma$-representations which are not type I. Further there exists a commutative group $G$ and a multiplier $\sigma$ such that $G$ has just one irreducible $\sigma$-representation and that one is infinite dimensional. $\mathrm{Cf}$. [16]. We now consider those modifications in the theory developed in the previous sections, which are needed to obtain, for a fixed multiplier $\sigma$, a general decomposition theory for $\sigma$-representations of $G$.

Let $G^{c, \sigma}$ denote the collection of concrete $\sigma$-representations which have a classical Hilbert space of $n$-tuples of complex numbers for a representation space. We give $G^{c, \sigma}$ a Borel structure exactly the way we gave $W^{c}$ a Borel structure. $\hat{G}^{\sigma}$ then denotes the collection of unitary equivalence classes of irreducible $\sigma$-representations of $G$ and we give $\hat{G}^{\sigma}$ a Borel structure in the same manner as for $\hat{G}$. We remark here that it has been shown in $[16, \S 3]$ that Theorems 8.1 through 8.6 of [15] remain true when $a^{c}$ and $\widehat{a}$ are replaced by $G^{c, \sigma}$ and $\hat{G}^{\sigma}$ respectively.

Let $G^{p, \sigma}$ denote the subset of $G^{c, \sigma}$ consisting of primary representations. Lemmas 2 and 3 of $\$ 2$ apply immediately to $\sigma$-representations where the space $Z$ is the set of all $\sigma$-representations $L$ of $G$ with a fixed representation space $\mathcal{H}$. Hence the reasoning of Theorem 1 may be used to show that $G^{p, \sigma}$ is a Borel subset of $G^{c, \sigma}$. Let $\widetilde{G}^{\sigma}$ denote the collection of all quasi-equivalence classes of primary $\sigma$-representations. Give $\tilde{G}^{\sigma}$ the Borel structure determined as a quotient of $G^{p, \sigma}$. Proposition 2 remains true if $w^{p}$ is replaced by $G^{p, \sigma}$. Indeed the given proof suffices for this case providing one takes into account the fact that Theorems 8.1 and 8.2 of [15] are true for $\sigma$-representations. It follows from this that every point of $\tilde{G}^{\sigma}$ is a Borel set. Propositions 3 and 4 and Theorem 2 , and their corollaries, all apply to $\sigma$-representations when $\tilde{\mathcal{W}}$ is replaced by $\widetilde{G}^{\sigma}$. The given proofs apply to this more general situation with only trivial modifications. The proof of Lemma 4 also applies to projective representations providing one notes that the proof of $[14$, Lemma 1.5] goes through for projective representations. Lemmas 5 and 7 of course have no meaning in this context as we have developed no terminology to deal with projective representations of $*$-algebras. For this reason the given proof of Proposition 5 cannot be used. We now prove an analogue of Lemma 7 which can be used to obtain the proper modification of the proof of Proposition 5.

LEMma $7^{\prime}$. Let $L$ denote a $\sigma$-representation of a separable locally compact group $G$. If $T \in Q(L)$, then there exists a sequence of operators $T_{i}$ of the form $T_{i}=\sum_{j=1}^{n_{i}} a_{i j} L_{x_{i j}}, i=1,2, \cdots$, where the $a_{i j}$ are complex numbers and $x_{i j} \in G$, $j=1, \cdots, n_{i}, i=1,2, \cdots$, such that $\left\|T_{i}\right\| \leqq\|T\|$ for all $i$ and the sequence $\left\{T_{i}\right\}$ converges to $T$ in the strong operator topology.

Proof. Let $a$ denote the *-algebra of all complex linear combinations of operators of the form $L_{x}$ where $x \in G$. Then by [1, Corollary 1 to Theorem 2 , p. 44], $Q$ is everywhere dense, in the strong topology, in $a(L)$, the von- 
Neumann algebra generated by the range of $L$. Suppose $T \in Q(L)$ and $m=\|T\|$. By Kaplansky's density theorem [1, Theorem 3, p. 46], the ball $Q_{m}$ of $Q$, of radius $m$, is everywhere dense, in the strong topology, in $Q_{m}$, the ball of $Q(L)$ of radius $m$. Thus $T$ is contained in the strong closure of $a_{m}$. By $[1$, corollary to Proposition 1, p. 33$]$, there exists a sequence of elements in $Q_{m}$ which converge strongly to $T$.

We leave it to the reader to make the necessary modifications in the proof of Proposition 5, using Lemma 7 ' in place of 7 , to make it apply to $\sigma$-representations. The rest of the decomposition theory now follows for projective representations, with only trivial modifications.

\section{REFERENCES}

1. J. Dixmier, Les algèbres d'operateurs dans l'espace Hilbertien (algèbres de von Neumann), Gauthier-Villars, Paris, 1957.

2. - Sur les structures Boréliennes $d u$ spectre d'une $C^{*}$-algèbre, Inst. Hautes Etudes Sci. Publ. Math. 6 (1960), 5-11.

3. - Dual et quasi-dual d'une algèbre de Banach involutive, Trans. Amer. Math. Soc. 104 (1962), 278-283.

4. J. Ernest, $A$ decomposition theory for unitary representations of locally compact groups, Bull. Amer. Math. Soc. 67 (1961), 385-388. 403.

5. J. M. G. Fell, The dual spaces of $C^{*}$-algebras, Trans. Amer. Math. Soc. 94 (1960), 365-

6. - $C^{*}$-algebras with smooth dual, Illinois J. Math. 4 (1960), 221-230.

7. J. Glimm, Type I C*-algebras, Ann. of Math. 73 (1961), 572-612.

8. A. Guichardet, Sur un problème posé par G. W. Mackey, C. R. Acad. Sci. Paris 250 (1960), 962-963.

9. P. R. Halmos, Introduction to Hilbert space and the theory of spectral multiplicity, Chelsea, New York, 1957.

10. C. Kuratowski, Topologie. I, 4th ed., Warsaw, 1958.

11. L. H. Loomis, Abstract harmonic analysis, D. Van Nostrand, New York, 1953.

12. G. W. Mackey, The theory of group representations, University of Chicago mimeographed notes, 1955.

13. - On induced representations of groups, Amer. J. Math. 73(1951), 576-592.

14. - Induced representations of locally compact groups. II. The Frobenius reciprocity theorem, Ann. of Math. 58 (1953), 193-221.

15. - Borel structure in groups and their duals, Trans. Amer. Math. Soc. 85 (1957), 134-165.

16. - Unitary representations of group extensions. I, Acta Math. 99 (1958), 265-311.

17. F. I. Mautner, Unitary representations of locally compact groups. I, Ann. of Math. 51 (1950), 1-25.

18. M. A. Naimark, Factor-representations of a locally compact group, Soviet Math. Dokl. 1 (1960), 1064-1066. 401-485.

19. J. von Neumann, On rings of operators. Reduction theory, Ann. of Math. 50 (1949),

20. I. E. Segal, Decompositions of operator algebras. I, II, Mem. Amer. Math. Soc. No. 9 (1951), 1-67 and 1-66.

The Institute for Advanced Study, Princeton, New Jersey

ThE UNIVERSITY OF Rochester, ROCHESTER, NEW York 